\title{
Current and emerging therapies for first line treatment of metastatic clear cell renal cell carcinoma
}

\author{
Michael T. Serzan, Michael B. Atkins \\ Georgetown Lombardi Comprehensive Cancer Center, Washington, DC 20057, USA.
}

Correspondence to: Dr. Michael B. Atkins, Department of Oncology, Georgetown Lombardi Comprehensive Cancer Center, 3970 Reservoir Rd, NW Research Building, Room E501, Washington, DC 20057, USA. E-mail: mba41@georgetown.edu

\begin{abstract}
How to cite this article: Serzan MT, Atkins MB. Current and emerging therapies for first line treatment of metastatic clear cell renal cell carcinoma. J Cancer Metastasis Treat 2021;7:39. https://dx.doi.org/10.20517/2394-4722.2021.76
\end{abstract}

Received: 26 Mar 2021 Accepted: 12 May 2021 Available online: 12 Jul 2021

Academic Editor: Hendrik Paul Van Poppel Copy Editor: Yue-Yue Zhang Production Editor: Yue-Yue Zhang

\begin{abstract}
The therapeutic landscape for advanced clear cell renal cell carcinoma (ccRCC) is rapidly evolving with improved knowledge of the biology of disease leading to the incorporation of a variety of antiangiogenic agents and immunotherapies. In this review, we discuss historical, current, and emerging first line treatment options for patients with advanced ccRCC. These include data with single agent vascular endothelial growth factor receptor tyrosine kinase inhibitors (TKIs): sunitinib, pazopanib and cabozantinib as well as the recently reported results for the combination of lenvatinib and everolimus (mTOR inhibitor). We also discuss results of the nivolumab antiprogrammed cell death (PD-1)/ipilimumab (anti-cytotoxic T lymphocyte-associated antigen 4) combination as well as emerging front-line data with nivolumab and pembrolizumab (anti-PD-1) monotherapy. Finally, we review data supporting recent approvals of TKI and anti-PD-1 or anti-PD-Ligand 1 (PD-L1) combinations (e.g., axitinib/pembrolizumab, axitinib/avelumab and cabozantinib/nivolumab) and initial outcomes of lenvatinib (multi-kinase inhibitor) and pembrolizumab. With many individual and combination treatment options and the lack of head-to-head comparisons, treatment selection will depend on the goals of therapy (endpoints) and the identification and validation of clinical and tumor-based predictive biomarkers that are linked to the desired treatment endpoints.
\end{abstract}

Keywords: Renal cell carcinoma, nivolumab, ipilimumab, axitinib, pembrolizumab, avelumab, biomarkers

The Author(s) 2021. Open Access This article is licensed under a Creative Commons Attribution 4.0 International License (https://creativecommons.org/licenses/by/4.0/), which permits unrestricted use, sharing, adaptation, distribution and reproduction in any medium or format, for any purpose, even commercially, as long as you give appropriate credit to the original author(s) and the source, provide a link to the Creative Commons license, and indicate if changes were made. 


\section{INTRODUCTION}

Kidney cancer is increasing in incidence worldwide with 403,000 new cases and 175,000 deaths annually based on the most recent GLOBOCAN statistics from $2018^{[1]}$. Renal cell carcinoma (RCC) is the most common form of kidney cancer, and is further classified by histologic subtypes with clear cell (cc) RCC being the most common (75\%) followed by papillary (10\%) and chromophobe $(5 \%)^{[2]}$. Localized RCC is typically managed with partial or radical nephrectomy associated with 5-year survival rates ranging from $70 \%$ to $90 \%$ depending on stage; however, up to $20 \%$ of such patients experience metastatic recurrence ${ }^{[3]}$. Approximately $20 \%$ of patients are diagnosed with metastatic disease at initial presentation. Metastatic clear cell renal cell carcinoma (ccRCC) historically carried a 5 -year survival rate of $13 \%{ }^{[4]}$. Advances in understanding the pathophysiology of RCC have elucidated the roles for targeted therapy against vascular endothelial growth factor receptors (VEGFR) with multi-kinase inhibitors, immune checkpoint inhibitors (ICI), and combination anti-VEGF and ICI regimens that have markedly improved outcomes.

ccRCC is near ubiquitously characterized by loss of heterozygosity of the von Hippel Lindau (VHL) gene (90\%) on chromosome $3 \mathrm{p} 8$ due to $V H L$ gene mutation (82\%) or epigenetic hypermethylation $(8 \%)^{[5-7]}$. Functional inactivation of the VHL tumor suppressor gene leads to accumulation of the transcription factor Hypoxia Inducible Factor-2 $\alpha$ in the absence of hypoxia. This accumulation serves as an oncoprotein driving several downstream pathways including VEGFA production leading to highly vascularized tumors ${ }^{[8,9]}$. Antiangiogenic tyrosine kinase inhibitors (TKI) of the VEGF pathway including sunitinib, pazopanib, and cabozantinib have improved outcomes in randomized clinical trials and are FDA approved therapies in the first line setting for metastatic RCC ${ }^{[10-13]}$. The Cancer Genome Atlas comprehensive genetic analyses have identified a subset of ccRCC patients with alterations in genes including MTOR (6\%), PTEN (4.3\%), and PIK3CA (2.9\%), leading to activation of the mechanistic target of rapamycin (mTOR) pathway ${ }^{[6]}$. Activation of this intracellular pathway leads to increased cell growth and division, thereby presenting biologic rationale for mTOR inhibition with everolimus and temsirolimus ${ }^{[14]}$.

Immunotherapy with checkpoint inhibitors (ICI) to the programmed cell death (PD-1) and cytotoxic T lymphocyte-associated antigen 4 (CTLA-4) pathways have also been investigated in patients with ccRCC. Nivolumab (anti-PD1) was approved in the second line for patients whose disease had progressed on antiangiogenic therapy based on the phase 3 Checkmate 025 study demonstrating overall survival (OS) and overall response rate (ORR) benefits compared to the mTOR inhibitor, everolimus ${ }^{[15]}$. The Checkmate 214 trial compared combination nivolumab and ipilimumab (anti-CLTA-4) to sunitinib for patients with treatment naïve advanced RCC. This study demonstrated significant improvement in OS and ORR favoring combination ICI therapy in the intermediate/poor risk (see below) populations, leading to FDA approval in April $2018^{[16]}$.

In this review, we discuss the recent and emerging first-line treatment options in ccRCC, with a focus on axitinib/pembrolizumab, axitinib/avelumab, cabozantinib/nivolumab, and lenvatinib/pembrolizumab and compare their efficacy to nivolumab/ipilimumab as well as VEGFR TKI and CPI monotherapy. We review safety and efficacy data and provide treatment recommendations based on clinical evidence and desired goals of therapy. In addition, we consider treatment sequencing and the need for biomarkers; we look to the future as novel combinations with immunotherapy backbones come to the forefront of the treatment paradigm.

\section{Clinical prognostic biomarkers}

The selection of first line treatment for patients with advanced ccRCC has been guided by risk stratification models developed by the Memorial Sloan Kettering Cancer Center (MSKCC) and the International 
Metastatic Renal Cell Carcinoma Database Consortium (IMDC ${ }^{[17,18]}$. The earlier MSKCC model was developed to predict benefit from interferon- $\alpha$, whereas the IMDC model predicted benefit from VEGFR TKI. Both models include time from diagnosis to treatment, Karnofsky performance status, and hemoglobin and calcium concentrations. Additionally, the MSKCC model incorporates lactate dehydrogenase level, whereas IMDC includes neutrophil and platelet count. In both models, patients with favorable-risk disease have 0 risk factors, those with intermediate-risk disease have 1-2 factors, and those with poor-risk disease have greater than 3 factors. The IMDC model has been utilized as a risk stratification tool for clinical trials of VEGFR TKI and combination regimens; however, its applicability to immunotherapy is likely limited. We will highlight potential future mostly laboratory biomarkers in development.

\section{FIRST LINE TREATMENT OPTIONS FOR CCRCC}

\section{Targeting angiogenesis and the VEGF pathway}

ccRCC is strongly associated with mutations in the VHL tumor suppressor gene, which results in functional inactivation of VHL proteins and downstream hypoxia-independent upregulation of pro-angiogenic factors including VEGF. Among all epithelial cancers, ccRCC has the highest expression of VEGFA, providing rationale for targeting VEGF and its receptor ${ }^{[19]}$. VEGF receptor blockade with TKIs in RCC has demonstrated several physiologic changes including reduction in blood vessel density, decreased tumor perfusion, and may lead to infarction of the VEGF-dependent tumor microenvironment ${ }^{[20]}$. Resistance to TKI therapy has been demonstrated to occur by angiogenic escape through activation of compensatory vascular signaling pathways including platelet derived growth factor (PDGFR), MET, AXL, and fibroblast growth factor receptor (FGFR) ${ }^{[21]}$. TKIs to multiple tyrosine kinases in addition to the VEGF receptor, including PDGFR (pazopanib, sunitinib, lenvatinib), MET/AXL (cabozantinib), FGFR (lenvatinib) were developed to simultaneously target parallel pathways causing decreased tumor vascularization and growth and delayed angiogenic escape $\mathrm{e}^{[22]}$.

Sunitinib and pazopanib were the first TKI to improve PFS in the first line setting compared to interferonalpha $(\mathrm{IFN} \alpha)$ and placebo, respectively ${ }^{[1,1,12]}$. Sunitinib was FDA approved for first line therapy of ccRCC in January 2006 followed by pazopanib in October 2009. These agents were compared in the phase 3 COMPARZ trial with pazopanib demonstrating noninferiority in PFS with similar OS in all IMDC risk groups $^{[12]}$. However, there were considerable differences in OS outcomes for each agent across IMDC groups: favorable risk 42.5 and 43.6 months, intermediate risk 26.9 and 26.1 months, and poor risk 9.9 and 7.7 months, respectively. Patients required frequent dose reductions (44\%-51\%) and discontinuation (20\%$24 \%$ ) due to adverse effects with similar grade 3-4 hypertension (15\%). Differences in safety and tolerability were noted with pazopanib demonstrating higher rates of liver function abnormalities and sunitinib higher rates of fatigue, palmar-plantar dysesthesia, and cytopenias. The phase $3 \mathrm{~b}$ PISCES sequential cross-over trial demonstrated superior patient and provider preference as well as higher health-related quality of life measures for pazopanib over sunitinib ${ }^{[23]}$. Pazopanib emerged as a preferred front line VEGFR TKI agent based on similar efficacy, less toxicity, and better tolerability.

Although the mTOR inhibitor temsirolimus was approved in the first line setting for patients with intermediate and poor risk RCC based on its superiority to interferon in the Global ARCC trial, the RECORD-3 trial subsequently showed that the oral mTOR inhibitor everolimus was inferior to sunitinib across all IMDC risk groups ${ }^{[24,25]}$. As a consequence, $\mathrm{mTOR}$ inhibitor use has been relegated to second or later lines of therapy, particularly in patients with tumors showing mutations in the PI3K, MTOR, TSC pathway or in combination with VEGFR TKI such as lenvatinib ${ }^{[26]}$. 
MET and AXL expression has been associated with aggressive disease and may mediate resistance to VEGFR TKI therapy ${ }^{[27]}$. The randomized phase 2 CABOSUN trial compared cabozantinib, an oral multi kinase inhibitor to VEFGR, MET, and AXL to sunitinib for treatment-naïve patients with intermediate/poor risk disease ${ }^{[13]}$. The trial met its primary endpoint of investigator assessed PFS (HR = 0.66; 95\%CI: 0.46-0.95; $P=0.012$ ) which was confirmed by independent radiology committee (IRC) with extended follow up (HR = 0.48; 95\%CI: 0.31-0.74, $P=0.0008)^{[28]}$. Further analysis demonstrated PFS benefit of cabozantinib over sunitinib across both IMDC risk groups, and regardless of tumor burden, metastatic site and MET expression status. Although there was a trend towards longer overall survival with cabozantinib 26.6 months vs. sunitinib 21.2 months ( $\mathrm{HR}=0.80 ; 95 \% \mathrm{CI}$ : 0.53-1.21), this study was underpowered to assess OS differences. Cabozantinib tolerance was similar to sunitinib with comparable rates of dose reduction (46\% vs. $35 \%)$ and discontinuation ( $21 \%$ vs. $22 \%)$. Also, common grade $3-4$ adverse events were similar between cabozantinib and sunitinib with hypertension ( $28 \%$ vs. $21 \%)$, fatigue (6\%vs. $17 \%)$, diarrhea (10\%vs. $11 \%)$, and thrombocytopenia ( $1 \% v s .11 \%)$. Based on the PFS benefit, cabozantinib was approved by the FDA in December 2017 for patients with intermediate/poor risk treatment naïve ccRCC.

Most recently, the phase 3 CLEAR study for patients with all-risk ccRCC compared first line sunitinib to lenvatinib/everolimus or lenvatinib/pembrolizumab ${ }^{[29]}$. The study met its primary endpoint of PFS by IRC for lenvatinib/everolimus compared to sunitinib ( $\mathrm{HR}=0.65 ; 95 \% \mathrm{CI}$ : 0.53-0.80). Despite this PFS benefit with higher ORR ( $54 \%$ vs $36 \%$ ) and CR rates ( $10 \%$ vs $4 \%$ ), there was no difference in overall survival (HR = 1.15; 95\%CI: 0.88-1.50). These results suggest that either the FGFR inhibition from lenvatinib or the addition of mTOR inhibition with everolimus may lead to enhanced initial antitumor response; however, this benefit may compromise the efficacy of subsequent therapy, thereby limiting the impact of this regimen on OS.

Despite the PFS benefits of sunitinib, pazopanib, cabozantinib, and lenvatinib/everolimus in the first line, these therapies were associated with frequent dose reductions (35\%-51\%) and rates of discontinuation due to adverse effects (20\%-24\%), many of which were postulated to be mediated by off-target inhibition of the PDGFR, KIT, and FLT-3 pathways. First line trials utilizing the more potent and selective second generation VEGFR TKIs axitinib and tivozanib compared to sorafenib were hypothesized to improve efficacy and reduce adverse effects. A phase 3 trial of first line axitinib compared to sorafenib showed numerical differences in PFS; however, it did not establish a statistically significant difference between the two treatments 10.1 months vs. 6.5 months $(\mathrm{HR}=0.77 ; 0.56-1.05)^{[30]}$. In addition, while axitinib showed significantly higher ORR $32 \%$ vs. $15 \%$ ( 1 sided $P=0.0006$ ), no difference in median OS 21.7 months vs. 23.3 months $(\mathrm{HR}=0.95 ; 0.73-1.36)$ compared to sorafenib was observed. One key limitation of this study was relatively small sample size $(N=288)$ to detect anything but a large magnitude of difference between therapies. The TIVO-1 study of first line tivozanib, a potent and selective TKI to VEGFR, c-Kit, and PDGFR compared to sorafenib met its primary endpoint of improved median PFS 11.9 months vs. 9.1 months (HR $=0.79 ; 0.63-0.99 ; P=0.042)^{[31]}$. However, OS analysis showed a trend toward longer survival on the sorafenib arm than on the tivozanib arm - median 29.3 months $v s .28 .8$ months $(H R=1.245 ; 0.95-1.62 ; P=0.105)$. These discordant PFS and OS results were hypothesized to be related to a greater proportion of patients in the sorafenib arm receiving next-line VEGFR TKI treatment ( $63 \%$ vs. $13 \%$ in the tivozanib arm) particularly with tivozanib (as part of the study). Although neither axitinib nor tivozanib were approved by the FDA for the first line setting, both of these agents have improved PFS compared to sorafenib in the second or later lines of therapy (leading to their FDA approval) and because of their improved therapeutic index related to their more selective targeting of the VEGF axis, they might offer advantages as backbones for combination regimens ${ }^{[32,33]}$. 
Antiangiogenic therapy with TKI greatly improved outcomes for patients with metastatic ccRCC relative to cytokine-based therapies. Despite consistent improvements in ORR and PFS, these regimens were not curative. Patients inevitably experienced progression of disease necessitating sequential switching to a different therapy, often another VEGFR TKI or an mTOR inhibitor. These successive agents increased the cumulative incidence of off-target adverse effects, which impacted quality of life and contributed substantial financial toxicity over time. As such, the current role of antiangiogenic TKI monotherapy is limited to patients who cannot receive ICI therapy due to active autoimmune disease or high dose steroids for central nervous system metastases.

\section{Immune checkpoint inhibitor therapies}

Initial evidence of immunogenicity in RCC was demonstrated in cytokine-based therapies with high dose interleukin 2 and IFN $\alpha$, which showed durable, complete responses in small subsets of patients ${ }^{[3,35]}$. The ability to induce an adaptive immune response relies on several aspects including tumor antigenicity, extent of immune cell infiltrate and immunomodulatory aspects within the tumor microenvironment ${ }^{[36]}$. ccRCC tumors are characterized by rich leukocyte infiltrates of CD8+ and CD4+ T cells as well as myeloid derived macrophages and neutrophils ${ }^{[37]}$. Tumors with an abundance of myeloid derived suppressor cells (MDSC) and polymorphonuclear leukocytes have been associated with higher tumor grade and shorter overall survival $^{[38]}$.

Checkpoint inhibitors are monoclonal antibodies that block physiologic or tumor cell mediated modulation of cellular immunity thereby restoring antigen specific cytotoxic $\mathrm{T}$ cell-mediated immune response ${ }^{[39]}$. Two critical checkpoints include interactions between CTLA receptor and its ligands CD80/86 on antigen presenting cells typically in peripheral immune organs and the PD-1 receptor and its ligands PD-L1/L2 in the tumor microenvironment. CTLA-4 binding to its ligand CD80/86 inhibits T cell activation. Therapeutic inhibition of this interaction with the CTLA-4 inhibitor ipilimumab leads to augmentation of T cell activation and proliferation of $\mathrm{T}$ cell subsets ${ }^{[40]}$. In the tumor microenvironment, tumor and immune cells can upregulate $\mathrm{PD}-\mathrm{L} 1 / \mathrm{L} 2$ ligands that bind the $\mathrm{PD}-1$ receptor on tumor reactive $\mathrm{T}$ cells leading to suppression of $\mathrm{T}$ cell activity ${ }^{[4]}$. Inhibition of this interaction with PD-1 antibodies (nivolumab, pembrolizumab) or PD-L1 antibodies (avelumab, atezolizumab) restores cytotoxic T cell activity and helper $\mathrm{T}$ cell cytokine production.

Nivolumab was the first ICI to show benefit in patients with advanced RCC. Nivolumab was compared to everolimus in patients who had exhibited disease progression on antiangiogenic therapy and showed improved ORR $25 \%$ vs. $5 \%$ and OS 25.0 months vs. 19.6 months $(\mathrm{HR}=0.73 ; 0.57-0.93 ; P=0.002)^{[15]}$. Activity relative to everolimus was particularly apparent in the MSKCC poor risk population (HR death $=0.47$; 0.30 0.73). Although these results were sufficient to confer FDA approval for nivolumab monotherapy, the efficacy was felt to be insufficient to be superior to VEGFR TKIs in treatment naïve patients. However, the Checkmate 016 trial explored the combination of nivolumab and ipilimumab in patients with either treatment naïve or VEGFR TKI resistant ccRCC and showed higher ORR (40.4\%) and median PFS (7.7 months) than had been observed with nivolumab monotherapy in the CM 025 trial suggesting it was more efficacious ${ }^{[42]}$.

As a consequence, combination nivolumab and ipilimumab was compared to sunitinib in treatment naïve patients in the Checkmate 214 phase 3 trial with co-primary endpoints OS, PFS, and ORR in the intermediate/poor risk disease group ${ }^{[17]}$. The study initially met two of three primary endpoints in its target population at median follow up 25.2 months. The combination demonstrated improved OS (HR death $=$ $0.63 ; P<0.001)$, ORR $42 \% v s .27 \%(P<0.001)$, and complete response rate $9 \% v s .1 \%$ relative to sunitinib 
[Table 1]. PFS was improved; however, it did not meet pre-specified level of significance $(\mathrm{HR}=0.82 ; P=$ 0.03). Subgroup analysis confirmed OS and ORR benefit regardless of PD-L1 tumor expression in intermediate/poor risk patients. However, patients with tumor PD-L1 >1\% demonstrated enhanced OS benefit from the combination immunotherapy (HR OS $=0.45$; 0.29-0.71) relative to those with tumor PD$\mathrm{L} 1<1 \%(\mathrm{HR}$ OS $=0.73$; 0.56-0.96). Patients with tumor PD-L1 $>1 \%$ demonstrated longer PFS with the combination relative to sunitinib (HR PFS $=0.46$; 0.31-0.67), whereas patients with tumor PD-L1 $<1 \%$ did $\operatorname{not}(\mathrm{HR}$ PFS $=1.00 ; 0.80-1.26)$.

In patients with favorable risk disease, sunitinib demonstrated significantly improved early outcomes relative the nivolumab/ipilimumab with ORR 52\% vs. 29\% $(P<0.001)$ and PFS 25.1 months vs. 15.3 months $(\mathrm{HR}=2.18 ; 1.29-3.68 ; P<0.001)$ that did not extend to $\mathrm{OS}(\mathrm{HR}=1.19 ; P=0.44)$. Interestingly, the efficacy of nivolumab and ipilimumab was similar in favorable risk patients compared with intermediate/poor risk patients with ORR $39 \%$ and $42 \%$, CR $8 \%$ and $11.3 \%$, and landmark 42 months PFS $28 \%$ and $35 \%{ }^{[43]}$. Furthermore, at 48 months there was a crossing of the Kaplan Meier OS curves between sunitinib and nivo/ipi indicating the potential for a OS benefit to eventually emerge favoring the combination ${ }^{[44]}$. Post hoc analysis of patients with aggressive sarcomatoid features, the vast majority of whom had intermediate or poor risk disease, showed remarkable benefits favoring nivolumab/ipilimumab to sunitinib in ORR 60.8\% vs. $23.1 \%$, CR rate $18.9 \%$ vs. $3.1 \%$, median PFS 26.5 months vs. 5.1 months $(\mathrm{HR}=0.54 ; 0.33-0.86 ; P=0.0093)$, and median OS not reached vs. 14.2 months $(\mathrm{HR}=0.45 ; 0.3-0.7 ; P=0.0004)^{[45]}$. Taken together, nivolumab/ipilimumab has emerged as the standard regimen for patients with intermediate/poor risk disease and those with sarcomatoid features. For patients with favorable risk disease, the early ORR and PFS benefits observed on sunitinib may be attributable to the relative efficacy of anti-angiogenic therapy in patients with less aggressive disease; however, maturing long term data of similar overall survival suggests a potential role for nivolumab/ipilimumab in this population.

Treatment related grade 3-4 adverse events were lower on nivolumab/ipilimumab (46\%) compared to sunitinib (63\%) [Table 2]. Nivolumab/ipilimumab was associated with high rate of immune related adverse events at $80 \%$ with $29 \%$ requiring high dose steroids for treatment. The discontinuation rate from all-cause adverse events was $22 \%$ for the combination and $13 \%$ for sunitinib. Interestingly, patients who discontinued nivolumab/ipilimumab treatment due to toxicity, exhibited a better OS than patients who did not experience treatment limiting toxicity. Patient reported outcomes of quality of life showed consistent mean change from baseline favoring combination therapy relative to sunitinib $(P<0.001)$ which was evident despite discontinuing QOL measurements at the time of treatment discontinuation even in patients continuing to exhibit long-term disease control.

The unprecedented improvements in overall survival with nivolumab/ipilimumab have led to efforts to investigate if subsets of patients could derive similar long-term benefits from single agent anti-PD-1 agents while avoiding toxicity of combination with ipilimumab. The Keynote 427 single-arm phase 2 study examined first line pembrolizumab monotherapy for patients with advanced RCC with the primary endpoint of ORR by blinded independent central review. Patients with ccRCC (Cohort A) demonstrated ORR 36.4\% with CR 2.7\%, median PFS 7.1 months, and median OS not reached at median follow up 18 months ${ }^{[46]}$. ORR was numerically higher for patients with intermediate/poor risk disease $39.7 \%$ compared to favorable risk $31.0 \%$ as well as patients with PD-L1 positive tumors $44.2 \%$ compared to $29.3 \%$ for those with PD-L1 negative tumors. Patients with sarcomatoid differentiation had an ORR of 63.6\%. Treatment related adverse effects occurred in $73.6 \%$ of patients with grade $3-5$ occurring in $18.2 \%$. These results show clinical activity of pembrolizumab monotherapy in the first line setting with perhaps increased benefit in intermediate/poor risk disease and sarcomatoid differentiation groups and lower rates of severe grade 3-5 


\begin{tabular}{|c|c|c|c|c|c|c|c|}
\hline \multirow[t]{2}{*}{ Study design/treatment } & \multirow{2}{*}{$\begin{array}{l}\text { Treatment } \\
\text { arms }\end{array}$} & \multicolumn{2}{|c|}{$\begin{array}{c}\text { ORR, CR (by IRC } \\
\text { unless otherwise } \\
\text { noted) }\end{array}$} & \multicolumn{2}{|c|}{$\begin{array}{l}\text { PFS (by IRC unless } \\
\text { otherwise noted) }\end{array}$} & \multicolumn{2}{|c|}{ os } \\
\hline & & $\begin{array}{l}\text { ORR\% } \\
\text { (CR) }\end{array}$ & $\begin{array}{l}\text { HR } \\
(95 \% \mathrm{Cl})\end{array}$ & $\begin{array}{l}\text { Median, } \\
\text { mo }\end{array}$ & $\begin{array}{l}\text { HR } \\
(95 \% \mathrm{Cl})\end{array}$ & $\begin{array}{l}\text { Median, } \\
\text { mo }\end{array}$ & $\begin{array}{l}\text { HR } \\
(95 \% \mathrm{Cl})\end{array}$ \\
\hline \multirow{5}{*}{$\begin{array}{l}\text { Checkmate } 214^{[16,43]} \\
-R \text {, ph } 3 \text {; treatment naïve patients stratified } \\
\text { by IMDC risk }(N=1096) \\
\text { - Co-Primary Endpoints: PFS, ORR, OS in } \\
\text { int/poor risk } \\
\text { - Nivo/lpi ( } 3 \mathrm{mg} / \mathrm{kg} \mathrm{Nivo} \mathrm{+} 1 \mathrm{mg} / \mathrm{kg} \text { Ipi q3wk } \\
\text { for } 4 \text { doses, then } 3 \mathrm{mg} / \mathrm{kg} \text { Nivo q2wk) vs. Sun } \\
\text { ( } 50 \mathrm{mg} \text { qd)a }\end{array}$} & $\begin{array}{l}\text { Int/Poor risk } \\
\text { Nivo/Ipi }(N= \\
425) \\
\text { Sun }(N=422)\end{array}$ & $\begin{array}{l}42.1 \% \\
(10.1) \\
26.3 \% \\
(1.4)\end{array}$ & $P<0.001$ & $\begin{array}{l}11.6 \\
8.3\end{array}$ & $\begin{array}{l}0.75(0.62- \\
0.90) \\
(P=0.015)\end{array}$ & $\begin{array}{l}47 \\
26.6\end{array}$ & $\begin{array}{l}0.66(0.55- \\
0.80) \\
(P< \\
0.0001)\end{array}$ \\
\hline & $\begin{array}{l}\text { Favorable risk } \\
\text { Nivo/Ipi }(N= \\
125) \\
\text { Sun }(N=124)\end{array}$ & $\begin{array}{l}28.8 \% \\
(12.8) \\
54.0 \% \\
(5.6)\end{array}$ & $P<0.0001$ & $\begin{array}{l}17.0 \\
28.8\end{array}$ & $\begin{array}{l}1.65(1.16- \\
2.35) \\
(P= \\
0.0049)\end{array}$ & $\begin{array}{l}N R \\
N R\end{array}$ & $\begin{array}{l}1.19(0.77- \\
1.85) \\
(P=0.43)\end{array}$ \\
\hline & $\begin{array}{l}\text { ITT } \\
\text { Nivo/Ipi }(N= \\
550) \\
\text { Sun }(N=546)\end{array}$ & $\begin{array}{l}39.1 \% \\
(10.7) \\
32.6 \% \\
(2.4)\end{array}$ & $P=0.02$ & $\begin{array}{l}12.4 \\
12.3\end{array}$ & $\begin{array}{l}0.88(0.75- \\
1.04) \\
(P=0.126)\end{array}$ & $\begin{array}{l}\text { NR } \\
38.4\end{array}$ & $\begin{array}{l}0.72(0.49- \\
0.95) \\
(P= \\
0.0002)\end{array}$ \\
\hline & $\begin{array}{l}\text { Int/Poor (PD-L } \\
<1) \\
\text { Nivo/Ipi }(N= \\
284) \\
\text { Sun }(N=278)\end{array}$ & $\begin{array}{l}37 \% \\
28 \%\end{array}$ & $P=0.03$ & $\begin{array}{l}11.0 \\
10.4\end{array}$ & $\begin{array}{l}1.00 \\
(0.8-1.26)\end{array}$ & $\begin{array}{l}\text { NR } \\
\text { NR }\end{array}$ & $\begin{array}{l}0.73(0.56- \\
0.96)\end{array}$ \\
\hline & $\begin{array}{l}\text { Int/Poor (PD-L } \\
>1) \\
\text { Nivo/Ipi }(N= \\
100) \\
\text { Sun }(N=114)\end{array}$ & $\begin{array}{l}58 \% \\
22 \%\end{array}$ & $P<0.001$ & $\begin{array}{l}22.8 \\
5.9\end{array}$ & $\begin{array}{l}0.46 \\
(0.31-0.67)\end{array}$ & $\begin{array}{l}N R \\
N R\end{array}$ & $\begin{array}{l}0.45(0.29- \\
0.71)\end{array}$ \\
\hline \multirow{3}{*}{$\begin{array}{l}\text { IMmotion } 151^{[58]} \\
\text { - R, ph 3; treatment naïve patients ( } N=915 \text { ) } \\
\text { - Co-Primary Endpoints: PFS (by inv) in PD- } \\
\text { L1+, OS in ITT } \\
\text { - Atezo/Bev (Atezo } 1200 \mathrm{mg} \text { IV q3wk + } 15 \\
\mathrm{mg} / \mathrm{kg} \text { Bev IV q3wk) vs. Sun (50 mg qd)a }\end{array}$} & $\begin{array}{l}\text { PD-L1+ } \\
\text { Atezo/Bev }(N= \\
178) \\
\text { Sun }(N=184)\end{array}$ & $\begin{array}{l}\text { Inv } \\
43 \%(9) \\
35 \%(4)\end{array}$ & - & $\begin{array}{l}\operatorname{lnv} \\
11.2 \\
7.7\end{array}$ & $\begin{array}{l}0.74(0.57- \\
096) \\
P=0.02\end{array}$ & $\begin{array}{l}34.0 \\
32.7\end{array}$ & $\begin{array}{l}0.84(0.62- \\
1.15) \\
P=0.28\end{array}$ \\
\hline & $\begin{array}{l}\text { PD-L1 }+ \\
\text { Atezo/Bev }(N= \\
178) \\
\text { Sun }(N=184)\end{array}$ & $\begin{array}{l}\text { IRC } \\
36 \%(15) \\
33 \%(8)\end{array}$ & - & $\begin{array}{l}\text { IRC } \\
8.9 \\
7.2\end{array}$ & $\begin{array}{l}0.93 \\
(0.72-1.21)\end{array}$ & - & - \\
\hline & $\begin{array}{l}\text { ITT } \\
\text { Atezo/Bev }(N= \\
454) \\
\text { Sun }(N=461)\end{array}$ & $\begin{array}{l}\text { Inv } \\
37 \%(5) \\
33 \%(2)\end{array}$ & - & $\begin{array}{l}\text { Inv } \\
11.2 \\
8.4\end{array}$ & $\begin{array}{l}0.83(0.70- \\
0.97) \\
P=0.02\end{array}$ & $\begin{array}{l}33.4 \\
34.9\end{array}$ & $\begin{array}{l}0.93(0.76- \\
1.14) \\
P=0.47\end{array}$ \\
\hline \multirow{3}{*}{$\begin{array}{l}\text { KEYNOTE } 426^{[59,60]} \\
\text { - R, open label ph 3; treatment naïve patients } \\
(N=861) \\
\text { - Co-Primary Endpoints: PFS (by ICR), OS in } \\
\text { ITT } \\
\text { - Axi/Pembro (Axi } 5 \mathrm{mg} \mathrm{PO} \mathrm{qd} \mathrm{+} \mathrm{Pembro} 200 \\
\text { g IV q3wk) vs. Sun ( } 50 \mathrm{mg} \text { qd)a }\end{array}$} & $\begin{array}{l}\text { ITT } \\
\text { Axi/Pembro (N } \\
=432) \\
\text { Sun }(N=429)\end{array}$ & $\begin{array}{l}60 \%(9) \\
40 \%(3)\end{array}$ & $P<0.0001$ & $\begin{array}{l}15.4 \\
11.1\end{array}$ & $\begin{array}{l}0.71(0.60- \\
0.84) \\
P<0.0001\end{array}$ & $\begin{array}{l}\text { NR } \\
35.7\end{array}$ & $\begin{array}{l}0.68(0.55- \\
0.85) \\
P=0.0003\end{array}$ \\
\hline & $\begin{array}{l}\text { Int/Poor risk } \\
\text { Axi/Pembro (N } \\
=294) \\
\text { Sun }(N=298)\end{array}$ & $\begin{array}{l}55.8 \%(8) \\
35.2 \%\end{array}$ & - & $\begin{array}{l}12.7 \\
8.3\end{array}$ & $\begin{array}{l}0.69(0.56- \\
0.84) \\
P=0.0002\end{array}$ & $\begin{array}{l}\text { NR } \\
28.9\end{array}$ & $\begin{array}{l}0.63(0.50- \\
0.81) \\
P=0.0001\end{array}$ \\
\hline & $\begin{array}{l}\text { Favorable Risk } \\
\text { Axi/Pembro ( } \\
=138) \\
\text { Sun }(N=131)\end{array}$ & $\begin{array}{l}69.6 \%(11) \\
50.4 \%(6)\end{array}$ & - & $\begin{array}{l}20.8 \\
18.0\end{array}$ & $\begin{array}{l}0.79(0.57- \\
1.09) \\
P=0.078\end{array}$ & $\begin{array}{l}\text { NR } \\
\text { NR }\end{array}$ & $\begin{array}{l}1.06(0.6- \\
1.86) \\
P=0.58\end{array}$ \\
\hline \multirow[t]{2}{*}{$\begin{array}{l}\text { JAVELIN Renal } 101^{[61,62]} \\
\text { - R, ph 3; treatment naïve patients ( } N=886 \text { ) } \\
\text { - Independent primary endpoints: PFS (by inv) } \\
\text { in PD-L1+, OS in PD-L1+ } \\
\text { - Axi/Avel (Axi } 5 \mathrm{mg} \mathrm{PO} \mathrm{qd+Avel} 10 \mathrm{mg} / \mathrm{kg} \\
\text { IV q2wk+ vs. Sun ( } 50 \mathrm{mg} \text { qd)a }\end{array}$} & $\begin{array}{l}\text { PD-L1+ } \\
\text { Axi/Avel }(N= \\
270) \\
\text { Sun }(N=290)\end{array}$ & $\begin{array}{l}\mathrm{BICR} \\
55.9 \% \\
(5.6) \\
27.2 \% \\
(2.4)\end{array}$ & $\begin{array}{l}\mathrm{OR}=3.389 \\
(2.34-4.90)\end{array}$ & $\begin{array}{l}\text { BICR } \\
13.8 \\
7.0\end{array}$ & $\begin{array}{l}0.62(0.49- \\
0.77) \\
P<0.0001\end{array}$ & $\begin{array}{l}\text { NR } \\
28.6\end{array}$ & $\begin{array}{l}0.83(0.59- \\
1.15) \\
P=0.13\end{array}$ \\
\hline & $\begin{array}{l}\text { ITT } \\
\text { Axi/Avel }(N= \\
442) \\
\text { Sun }(N=444)\end{array}$ & $\begin{array}{l}\text { BICR } \\
52.5 \% \\
(3.8) \\
27.3 \% \\
(2.0)\end{array}$ & $\begin{array}{l}\mathrm{OR}=2.99 \\
(2.23-3.99)\end{array}$ & $\begin{array}{l}\text { BICR } \\
13.8 \\
8.4\end{array}$ & $\begin{array}{l}0.69(0.57- \\
0.82) \\
P<0.0001\end{array}$ & $\begin{array}{l}\text { NR } \\
\text { NR }\end{array}$ & $\begin{array}{l}0.80(0.61- \\
1.02) \\
P=0.039\end{array}$ \\
\hline $\begin{array}{l}\text { Checkmate } 9 E R^{[63]} \\
-R \text {, open label ph 3; treatment naïve patients } \\
(N=651) \\
\text { - Primary endpoints: PFS (BICR) in ITT }\end{array}$ & $\begin{array}{l}\text { ITT } \\
\text { Cabo/Nivo }(N= \\
323) \\
\text { Sun }(N=328)\end{array}$ & $\begin{array}{l}55.7 \%(8) \\
27.1 \%(5)\end{array}$ & $P<0.0001$ & $\begin{array}{l}16.6 \\
8.3\end{array}$ & $\begin{array}{l}0.51(0.41- \\
0.64) \\
P<0.0001\end{array}$ & $\begin{array}{l}\text { NR } \\
\text { NR }\end{array}$ & $\begin{array}{l}0.60 \\
(0.40-89) \\
P=0.001\end{array}$ \\
\hline
\end{tabular}


mg IV q2wk vs. Sun (50 mg qd)a

\begin{tabular}{|c|c|c|c|c|c|c|c|}
\hline \multirow{3}{*}{$\begin{array}{l}\text { CLEAR }^{[29]} \\
-R_{\text {, open label ph 3; treatment naïve patients }} \\
(N=1069) \\
\text { - Primary Endpoint: PFS (by IRC) in ITT } \\
\text { - Lenv/Pembro (Lenv } 18 \mathrm{mg} \text { PO qd + Pembro } \\
200 \mathrm{mg} \text { IV q3wk vs. Lenv } 14 \mathrm{mg} \text { PO qd + Evero } \\
5 \mathrm{mg} \text { Po qd vs. Sun ( } 50 \mathrm{mg} \text { qd)a }\end{array}$} & $\begin{array}{l}\text { ITT } \\
\text { Lenv/Pembro( } \\
N=454)\end{array}$ & $71 \%$ (16) & $P<0.001$ & 23.9 & $\begin{array}{l}0.39(0.32- \\
0.49) \\
P<0.001\end{array}$ & $N R^{b}$ & $\begin{array}{l}0.66(0.49- \\
0.88) \\
P=0.005\end{array}$ \\
\hline & $\begin{array}{l}\text { Lenv/Evero ( } N \\
=461)\end{array}$ & $53 \%(10)$ & $P<0.001$ & 14.7 & $\begin{array}{l}0.65(0.53- \\
0.80) \\
P<0.001\end{array}$ & $N R^{b}$ & $\begin{array}{l}1.15(0.88- \\
1.5) \\
P=0.30\end{array}$ \\
\hline & $\operatorname{Sun}(N=461)$ & $36 \%(4)$ & & 9.2 & & $N R^{b}$ & \\
\hline
\end{tabular}

Atezo: Atezolizumab; Avel: avelumab; Axi: axitinib; Bev: bevacizumab; Cabo: cabozantinib; Cl: confidence interval; Evero: everolimus; HR: hazard ratio; IMDC: International Metastatic Renal Cell Carcinoma; int: intermediate; inv: investigator; Ipi: ipilimumab; IRC: independent review committee; ITT: intention to treat; Lenv: lenvatinib; mo: month(s); NA: not applicable; Nivo: nivolumab; NR: not reached; ORR: objective response rate; OS: overall survival; PD-L1: programmed cell death ligand 1; PFS: progression free survival; Ph: phase; q3wk: every 3 weeks; qd: once daily; r: randomized; Sun: sunitinib; wk: week.

Table 2. Hazard ratio over time in key studies

\begin{tabular}{|c|c|c|c|c|c|c|}
\hline \multirow[b]{3}{*}{ Median follow-up } & \multicolumn{6}{|c|}{ Checkmate $214^{[16,43]}$} \\
\hline & \multicolumn{2}{|c|}{ Favorable risk } & \multicolumn{2}{|c|}{ Intermediate/poor risk } & \multicolumn{2}{|c|}{ ITT } \\
\hline & PFS (HR) & OS (HR) & PFS (HR) & OS (HR) & PFS (HR) & OS (HR) \\
\hline $25.2 \mathrm{mo}$ & $\begin{array}{l}2.18(1.29-3.68) \\
(P<0.001)\end{array}$ & $\begin{array}{l}1.45(0.51-4.12) \\
(P=0.27)\end{array}$ & $\begin{array}{l}0.82(0.64-1.05) \\
(P=0.03)\end{array}$ & $\begin{array}{l}0.63(0.44-0.89) \\
(P<0.001)\end{array}$ & $\begin{array}{l}0.98(0.79-1.23) \\
(P=0.85)\end{array}$ & $\begin{array}{l}0.68(0.49-0.95) \\
(P<0.001)\end{array}$ \\
\hline $32.4 \mathrm{mo}$ & $\begin{array}{l}1.23(0.9-1.69) \\
(P=0.19)\end{array}$ & $\begin{array}{l}1.22(0.73-2.04) \\
(P=0.44)\end{array}$ & $\begin{array}{l}0.77(0.65-0.90) \\
(P<0.01)\end{array}$ & $\begin{array}{l}0.66(0.54-0.80) \\
(P<0.0001)\end{array}$ & $\begin{array}{l}0.85(0.73-0.98) \\
(P=0.03)\end{array}$ & $\begin{array}{l}0.71(0.59-0.86) \\
(P<0.01)\end{array}$ \\
\hline \multirow[t]{3}{*}{$43.6 \mathrm{mo}$} & $\begin{array}{l}1.65(1.16-2.35) \\
(0.0049)\end{array}$ & $\begin{array}{l}1.19(0.77-1.85) \\
(P=0.43)\end{array}$ & $\begin{array}{l}0.75(0.62-0.90) \\
(P=0.015)\end{array}$ & $\begin{array}{l}0.66(0.55-0.80) \\
(P<0.0001)\end{array}$ & $\begin{array}{l}0.88(0.75-1.04) \\
(P=0.126)\end{array}$ & $\begin{array}{l}0.72(0.61-0.86) \\
(P=0.0002)\end{array}$ \\
\hline & \multicolumn{6}{|l|}{ Keynote $426^{[59,60]}$} \\
\hline & \multicolumn{2}{|l|}{ Favorable risk } & \multicolumn{2}{|c|}{ Intermediate (int)/poor risk } & \multicolumn{2}{|l|}{ ITT } \\
\hline Median follow-up & PFS (HR) & OS (HR) & PFS (HR) & OS (HR) & PFS (HR) & OS (HR) \\
\hline $12.8 \mathrm{mo}$ & $0.81(0.53-1.24)$ & $0.64(0.24-1.68)$ & $\begin{array}{l}0.70 \text { (0.54-0.91) (int) } \\
0.58(0.35-0.94) \text { (poor) }\end{array}$ & $\begin{array}{l}0.53(0.35-0.82) \text { (int) } \\
0.43(0.23-0.81) \text { (poor) }\end{array}$ & $\begin{array}{l}0.69(0.57-0.84) \\
P<0.001\end{array}$ & $\begin{array}{l}0.53(0.38-0.74) \\
P<0.001\end{array}$ \\
\hline \multirow[t]{3}{*}{$30.6 \mathrm{mo}$} & $\begin{array}{l}0.79(0.57-1.09) \\
(P=0.078)\end{array}$ & $\begin{array}{l}1.06(0.6-1.86) \\
P=0.58\end{array}$ & $\begin{array}{l}0.69(0.56-0.84) \\
(P=0.0002)\end{array}$ & $\begin{array}{l}0.63(0.5-0.81) \\
(P=0.0001)\end{array}$ & $\begin{array}{l}0.71(0.60-0.84) \\
(P<0.0001)\end{array}$ & $\begin{array}{l}0.68(0.55-0.85) \\
P=0.0003\end{array}$ \\
\hline & \multicolumn{6}{|c|}{ Javelin Renal $101^{[61,62]}$} \\
\hline & PD-L1+ & & ITT & & & \\
\hline Median follow-up & PFS (HR) & OS (HR) & PFS (HR) & OS (HR) & & \\
\hline $11.6 \mathrm{mo}$ & $\begin{array}{l}0.61(0.47-0.79) \\
P<0.001\end{array}$ & $\begin{array}{l}0.82(0.53-1.28) \\
P=0.38\end{array}$ & $\begin{array}{l}0.69(0.56-0.84) \\
(P<0.01)\end{array}$ & $\begin{array}{l}0.69(0.56-0.84) \\
(P<0.001)\end{array}$ & & \\
\hline $19.2 \mathrm{mo}$ & $\begin{array}{l}0.62(0.49-0.77) \\
(P=0.0001)\end{array}$ & $\begin{array}{l}0.83(0.59-1.15) \\
(P=0.13)\end{array}$ & $\begin{array}{l}0.69(0.57-0.82) \\
(P<0.0001)\end{array}$ & $\begin{array}{l}0.80(0.61-1.02) \\
(P=0.03)\end{array}$ & & \\
\hline
\end{tabular}

HR: Hazard ratio; PFS: progression free survival; ITT: intention to treat; OS: overall survival; PD-L1: programmed cell death ligand 1; mo: month(s).

toxicity relative to nivolumab/ipilimumab.

Similar efforts have been made to investigate both anti-PD-L1 monotherapy in the first line setting and the feasibility of salvage nivolumab/ipilimumab. The HCRN GU 16-260 phase II trial treated patients with advanced ccRCC with first line nivolumab (Part A) with the primary endpoint of $\mathrm{ORR}^{[47]}$. Patients who experienced either progression of disease (PD) or stable disease (SD) at 48 weeks were eligible to receive salvage nivolumab/ipilimumab (Part B). In the total population, ORR was $31.7 \%$ with CR $5.7 \%$ with subgroup analysis showing patients with favorable risk disease had an ORR of 50\%, intermediate/poor risk disease had ORR of $25 \%$ and those with sarcomatoid tumors had an ORR of $31.8 \%$. The median duration of response was 19.3 months and median PFS was 7.4 months. Sixty patients were potentially eligible for 
salvage nivolumab/ipilimumab (Part B); however, 28 did not enroll due to symptomatic progression of disease (17), grade 3-4 toxicity on nivolumab (8), or other (3). Of the patients who received salvage therapy, best response was PR (13\%), SD (30\%), and PD (59\%). Grade 3-5 treatment-related adverse effects were seen in $28 \%$ on nivolumab monotherapy and $33 \%$ on nivolumab/ipilimumab. These results suggest a potential role for anti-PD-1 monotherapy in patients who have contraindications or an aversion to either an ipilimumab or VEGFR TKI containing combination regimen, particularly those with favorable risk disease. However, anti-PD-1 monotherapy is likely inferior to nivolumab/ipilimumab in patients with intermediate/poor risk disease - a question that is being formally addressed in the Checkmate 8Y8 (NCT03873402) protocol which is currently ongoing, or those whose tumors express sarcomatoid features ${ }^{[48]}$.

\section{Combination VEGF TKI and PD-1 therapy}

Angiogenic agents targeting VEGF and ICI therapies have improved survival for patients with advanced ccRCC and are standard therapies in the management of this disease. Combinations of antiangiogenic and ICI therapies have the potential to target distinct and complementary pathways, providing synergistic benefit with concurrent therapy compared with additive effects of sequential therapy. Recent preclinical studies have demonstrated that VEGFR TKI therapy alleviates immunosuppression in the tumor microenvironment through targeting regulatory T-cells and MDSC, promoting T-cell infiltration, and enhancing T-cell mediated cytotoxicity ${ }^{[4-52]}$. In vivo evidence from animal models has further shown that combination of sunitinib or cabozantinib with chimeric antigen receptor-modified $\mathrm{T}$ cells can increase antitumor efficacy and prolong survival compared to immunotherapy alone ${ }^{[53]}$. However, there is also preclinical evidence suggesting that anti-angiogenic therapy may have an antagonistic effect on the immune response, particularly in ccRCC, by increasing hypoxia in the TME thereby diminishing anti-tumor immunity and by upregulating CXCR4 expression leading to the influx of tumor-infiltrating regulatory $\mathrm{T}$ cells and MDSC ${ }^{[54-56]}$.

Early phase I evaluation of combination sunitinib or pazopanib with nivolumab or pembrolizumab for advanced RCC showed high rates of response; however, high-grade toxicities limited further investigation $^{[57]}$. More recently, several trials have investigated various antiangiogenic agents combined with anti-PD1/PD-L1 therapies compared to sunitinib alone. The IMmotion 151 phase 3 trial of first line combination atezolizumab (anti-PD-L1) and bevacizumab (monoclonal antibody against VEGF) was compared to sunitinib with co-primary endpoints PFS in PD-L1+ tumors and OS in overall population ${ }^{[58]}$. In patients with PD-L1+ tumors, the combination demonstrated significantly improved PFS (investigator assessed) compared to sunitinib alone (HR PFS $=0.74 ; 0.57-0.96 ; P=0.0217$ ) [Table 1]. In the overall population, there was no significant difference in PFS or OS between atezolizumab plus bevacizumab and sunitinib. On further examination by an IRC, patients with PD-L1+ tumors demonstrated similar PFS between the combination and sunitinib (HS PFS $=0.93$; 0.72-1.21). Interestingly, the IRC analysis of patients with PD-L1 negative tumors demonstrated a trend towards longer PFS in the atezolizumab plus bevacizumab groups compared to sunitinib; suggesting that either PD-L1 status is a poor predictive biomarker for response to the combination or that the Ventana SP142 assay scoring immune cell PD-L1 positivity may be a suboptimal assay. Atezolizumab and bevacizumab was well tolerated compared to sunitinib with lower rates of grade 3-4 treatment-emergent adverse events ( $40 \% v s$. 54\%) with $16 \%$ patients on atezolizumab and bevacizumab requiring corticosteroids for IRAE [Table 2]. Due to discordant PFS between investigator and IRC assessments and the absence of OS benefit, the combination of atezolizumab and bevacizumab was not approved for first line use. 
In contrast, two unique combinations of axitinib with either pembrolizumab or avelumab (anti-PD-L1) have demonstrated PFS benefits relative to sunitinib. The Keynote 426 phase 3 trial for first line ccRCC randomized patients to axitinib/pembrolizumab or sunitinib with co-primary endpoints of OS and PFS in the intention-to-treat population ${ }^{[5]}$. At first interim analysis at a median follow up 12.8 months, the combination demonstrated improvements in risk of death (HR OS $=0.53 ; 0.38-0.74 ; P<0.0001$ ), risk of progression $(\mathrm{HR} P F S=0.69 ; 0.57-0.84 ; P<0.001)$, and ORR (59.3\% vs. 35.7\%; $P<0.001)$ relative to sunitinib [Table 1]. The PFS and OS benefits were observed across all IMDC risk groups and PD-L1 expression categories. In a subsequent analysis at median follow up 27 months, the PFS benefit was maintained ( $\mathrm{HR}=$ 0.71 ), but the OS benefit was reduced (HR OS $=0.68$; 055-0.85; $P<0.001$ ) and was no longer apparent for the favorable risk population (HR OS $=1.06 ; 0.60-1.86)^{[60]}$. Axitinib/pembrolizumab had similar rates of grade 3-4 treatment-related adverse events compared to sunitinib at $67 \%$ and $62 \%$; however, the combination had higher rates of grade 3-4 liver enzyme elevation $7 \%-12 \% v s .2 \%-3 \%$, respectively [Table 2]. These results led to FDA approval of axitinib plus pembrolizumab for all-risk patients with advanced RCC in April 2019.

Axitinib combined with avelumab was compared to sunitinib in the Javelin Renal 101 phase 3 trial for first line ccRCC with independent primary endpoints of OS and PFS in patients with PD-L1 positive tumors ${ }^{[61]}$. In the $\mathrm{PD}-\mathrm{L} 1$ positive group, axitinib and avelumab demonstrated improvements in $\mathrm{PFS}$ ( $\mathrm{HR}=0.62$; 0.49$0.78 ; P<0.001$ ) and ORR $55.9 \%$ vs. $27.2 \%$ relative to sunitinib [Table 1]. These benefits of the combination were also demonstrated in the overall study population PFS $(\mathrm{HR}=0.69 ; 0.56-0.84 ; P<0.001)$ and ORR $52.5 \%$ vs. $27.3 \%{ }^{[62]}$. Axitinib and avelumab was well tolerated compared to sunitinib with similar rates of grade 3-4 treatment-emergent adverse events ( $71.2 \% v s .71 .5 \%)$ with $11 \%$ of combination therapy patients requiring corticosteroids for IRAE [Table 2]. These results led to FDA approval of axitinib plus avelumab for all-risk patients with advanced RCC in May 2019.

Two additional trials utilizing combinations of antiangiogenics with anti-PD-1 agents have resulted within the past year. In the Checkmate9ER phase 3 trial patients with treatment naïve ccRCC were randomized to cabozantinib/nivolumab or sunitinib with the primary endpoint of PFS by blinded independent central review $^{[63]}$. At first interim analysis of median follow up 18.1 months, the combination demonstrated improvements in PFS (HR $=0.51 ; 0.40-0.64 ; P<0.0001)$ and $\mathrm{OS}(\mathrm{HR}=0.60 ; 0.40-0.89 ; P<0.001)$ relative to sunitinib [Table 1]. The PFS and OS benefits were observed across all IMDC risk groups and $P D-L 1$ expression categories leading to FDA approval in January 2021. Cabozantinib/nivolumab had similar rates of grade 3-4 treatment-related adverse effects compared to sunitinib at $61 \%$ and $51 \%$ with $16 \%$ of patients on the combination requiring corticosteroids for IRAE [Table 2]. The CLEAR phase 3 trial for first line ccRCC randomized patients to sunitinib, lenvatinib/everolimus, or lenvatinib/pembrolizumab with primary endpoint PFS by IRC per RECIST1.1 ${ }^{[29]}$. At first interim analysis at a median follow up of 27 months, lenvatinib/pembrolizumab was superior to sunitinib in PFS ( $\mathrm{HR}=0.39 ; 0.32-0.49 ; P<0.001)$ and $\mathrm{OS}(\mathrm{HR}=$ $0.60 ; 0.40-0.89 ; P=0.001)$. Lenvatinib/pembrolizumab also had high rates of grade $3-4$ treatment-related adverse events ( $82 \%)$ necessitating dose reduction of lenvatinib in $68 \%$ and discontinuation of the combination in $13 \%$ of patients. Both cabozantinib/nivolumab and lenvatinib/pembrolizumab achieved high response rates as well as significant PFS and overall survival benefits for the intent to treat population. However, the contribution of cabozantinib and lenvatinib as more efficacious TKIs relative to sunitinib may confound these early results. Furthermore, the ability of these regimens to produce long-term plateauing of the PFS curve as well as their impact on OS over time will be of particular interest given the more limited effective treatment options following disease progression on regimens involving the use of more potent TKIs in the front-line setting. 
Combinations of anti-angiogenics with anti-PD-1/PD-L1 have been tolerable with common adverse effects being fatigue, hypertension, and diarrhea similar to adverse effects observed with sunitinib alone. However, higher rates of hypothyroidism (22\%-35\%) and grade 3-4 liver enzyme elevations (6\%-13\%) were observed in axitinib/pembrolizumab and axitinib/avelumab than with sunitinib. These warrant close monitoring as antiangiogenic and ICI therapies may potentiate synergistic or additive adverse effects that accumulate over time. Current trials of combination angiogenic and ICI therapies are evaluating patient-reported outcomes to determine in depth health-related quality of life over time while on therapy.

\section{Novel endpoints}

Over the past three years, pivotal trials of anti-PD-1 therapy combined with either anti-CTLA or various VEGFR TKIs have led to unprecedented improvements in outcomes for patients with advanced RCC. However, in the absence of head-to-head comparison, the optimal choice for first line therapy remains a debated issue as these regimens have yielded important differences in some novel endpoints. Anti-PD1/VEGFR TKI combinations have demonstrated improvements in "early" endpoints including ORR, PFS, and $<2$-year OS rates, perhaps favoring use in patients with symptomatic disease with the goal of more immediate disease control. Whereas, anti-PD-1/anti-CTLA therapy has led to more durable "late" endpoints including landmark PFS/OS, > 2-year OS rates, treatment free survival (TFS), and patientreported quality of life, suggesting greater benefit in sustained disease control.

As data from the Checkmate-214 study continue to mature with a minimum of 4-year follow up, the durable and late benefits have become more pronounced with PFS and OS plateaus greater than $30 \%$ and $50 \%$, respectively. The overall survival benefits for the ITT population on nivolumab/ipilimumab relative to sunitinib in the Checkmate 214 study have remained stable over time with initial median follow-up of 25 months $($ HR OS $=0.68 ; P<0.001)$ and interval follow-up at median 43 months $($ HR OS $=0.72 ; 0.61-0.86 ; P$ $=0.0002$ ). Furthermore, Regan et al.$^{[64,65]}$ analyzed 42 -month TFS, defined as time from protocol therapy cessation to time of subsequent systemic therapy or death. In the nivolumab/ipilimumab arm, $56 \%$ of patients were alive, $13 \%$ were on nivolumab maintenance, and $31 \%$ were surviving free of subsequent therapy. In the sunitinib arm, $47 \%$ of patients were alive, $7 \%$ remained on sunitinib therapy, and $12 \%$ were surviving free of subsequent treatment. The overall 42 -month restricted mean TFS was 7.8 months for nivolumab/ipilimumab and 3.3 months for sunitinib. Mean TFS for nivolumab/ipilimumab compared to sunitinib was three times as long in favorable risk patients (11.0 months $v s .3 .7$ months) and over twice as long in intermediate/poor risk patients (6.9 months vs. 3.1 months). These results highlight that patients across all IMDC risk groups experienced benefit from ICI therapy with greater survival time treatment-free without toxicity relative to sunitinib. Although nivolumab/ipilimumab carries a high risk of early immune related adverse events, many of these are reversible with short-term immunosuppression without evidence of detriment to anti-tumor immune effect. Based on its ability to produce robust and sustained anti-tumor responses with prolonged treatment free intervals, nivolumab/ipilimumab represents an excellent treatment choice for many patients with advanced ccRCC.

Several anti-PD-1/VEGFR TKI combinations have demonstrated substantial benefit in early endpoints of ORR 52\%-71\% and median PFS 13-24 months in the ITT populations. However, the ability of these regimens to extend early response benefits into durable long-term outcomes with PFS and OS plateaus remains to be established. Axitinib/pembrolizumab showed remarkable early overall survival benefit in ITT population at a median 12-month follow up (OS HR $=0.53$; 0.38-0.74; $P<0.001)$; however, this OS benefit appears to diminish at the median follow-up of 30 months (OS HR $=0.68 ; 0.55-0.85 ; P=0.0003$ ) [Table 3]. Similarly, axitinib/avelumab showed early overall survival benefit in ITT at the median follow-up of 12 months (OS HR $=0.69 ; 0.56-0.84 ; P<0.001$ ); however, this OS benefit appears to diminish at the median follow-up of 19 months (OS HR $=0.80 ; 0.61-1.02 ; P=0.03$ ) [Table 3]. The decreasing OS benefits in these 
Table 3. Safety data from key studies

\begin{tabular}{|c|c|c|c|c|c|c|c|c|c|c|c|c|c|}
\hline & \multicolumn{2}{|c|}{ Checkmate $214^{[43]}$} & \multicolumn{2}{|c|}{ 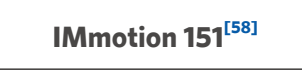 } & \multicolumn{2}{|c|}{ KEYNOTE $426^{[59,60]}$} & \multicolumn{2}{|c|}{$\begin{array}{c}\text { JAVELIN renal } \\
101^{16,, 62\}}\end{array}$} & \multicolumn{2}{|c|}{ Checkmate $9 \mathrm{ER}^{[63]}$} & \multicolumn{3}{|c|}{ CLEAR $^{[29]}$} \\
\hline & $\begin{array}{l}\text { Nivo/Ipi ( } \\
N=547)\end{array}$ & $\begin{array}{l}\text { Sun }(N \\
=535)\end{array}$ & $\begin{array}{c}\text { Atezo/Bev ( } \\
N=454)\end{array}$ & $\begin{array}{l}\text { Sun }(N \\
=461)\end{array}$ & $\begin{array}{c}\text { Axi/Pembro ( } \\
N=432)\end{array}$ & $\begin{array}{l}\text { Sun }(N \\
=429)\end{array}$ & $\begin{array}{c}\text { Axi/Avel ( } \\
N=442)\end{array}$ & $\begin{array}{l}\text { Sun }(N \\
=444)\end{array}$ & $\begin{array}{c}\text { Cabo/Nivo ( } \\
\boldsymbol{N}=\mathbf{3 2 3})\end{array}$ & $\begin{array}{l}\text { Sun }(N \\
=328)\end{array}$ & $\begin{array}{c}\text { Lenv/Pembro ( } \\
N=454)\end{array}$ & $\begin{array}{c}\text { Lenv/Evero ( } \\
N=461)\end{array}$ & $\begin{array}{l}\text { Sun }(N \\
=461)\end{array}$ \\
\hline Dose reductions, \% & - & 53 & - & 37 & 20 & 28 & 42 & 43 & 56 & 52 & 68 & 73 & 50 \\
\hline $\begin{array}{l}\text { AE leading to discontinuation } \\
\text { of entire treatment regimen, } \\
\%\end{array}$ & 22 & 13 & 5 & 8 & 7 & 12 & 8 & 13 & 5.6 & 17 & 13 & 19 & 14 \\
\hline Treatment-related deaths, $n$ & 8 & 4 & 5 & 1 & 4 & 7 & 3 & 1 & 1 & 2 & 4 & 3 & 1 \\
\hline $\begin{array}{l}\text { Treatment-emergent AE, } \\
\text { grades 3-4\%a }\end{array}$ & 47 & 64 & 40 & 54 & 67 & 62 & 71 & 71 & 61 & 51 & 82 & 83 & 72 \\
\hline Corticosteroids for IRAE, \%a & 29 & - & 16 & - & NR & - & 11 & - & 16 & - & NR & - & - \\
\hline \multicolumn{14}{|l|}{ Grade 3/4 AEs, \% } \\
\hline Hypertension & $<1$ & 16 & 14 & 17 & 21 & 18 & 26 & 17 & 13 & 13 & 28 & 23 & 19 \\
\hline Fatigue & 4 & 9 & 1 & 5 & 2 & 5 & 4 & 4 & 3 & 5 & 4 & 8 & 4 \\
\hline Diarrhea & 4 & 5 & 2 & 4 & 7 & 5 & 7 & 3 & 7 & 4 & 10 & 12 & 5 \\
\hline PPE & 0 & 9 & 0 & 9 & 5 & 4 & 6 & 4 & 8 & 8 & 4 & 3 & 4 \\
\hline Weight Loss & - & - & - & - & - & - & 3 & 1 & 1 & 0 & 8 & 7 & $<1$ \\
\hline Decreased appetite & 1 & 1 & $<1$ & 2 & 2 & $<1$ & 2 & 1 & 2 & 1 & 4 & 6 & 2 \\
\hline Proteinuria & - & - & 3 & $<1$ & 3 & 3 & - & - & 3 & 2 & 8 & 8 & 3 \\
\hline \multicolumn{14}{|l|}{$\begin{array}{l}\text { Grade 3/4 laboratory } \\
\text { abnormalities, } \%\end{array}$} \\
\hline Hypothyrodiism & $<1$ & $<1$ & $<1$ & $<1$ & $<1$ & 0 & $<1$ & $<1$ & $<1$ & $<1$ & 1 & 1 & 0 \\
\hline Increased lipase & 10 & 7 & - & - & - & - & - & - & 6 & 5 & 13 & 4 & 9 \\
\hline Neutropenia & - & - & $<1$ & 4 & $<1$ & 7 & $<1$ & 8 & $<1$ & 4 & 1 & 1 & 6 \\
\hline Anemia & 0 & 4 & $<1$ & 4 & $<1$ & 3 & 2 & 8 & 2 & 4 & 2 & 4 & 5 \\
\hline Thrombocytopenia & 0 & 5 & 1 & 5 & 0 & 5 & $<1$ & 6 & $<1$ & 5 & 1 & 4 & 6 \\
\hline Increased ALT & 5 & 2 & - & - & 12 & 3 & 6 & 3 & 5 & 2 & 4 & 3 & 2 \\
\hline Increased AST & 4 & 1 & - & - & 7 & 2 & 4 & 2 & 3 & 1 & 3 & 2 & 1 \\
\hline
\end{tabular}

${ }^{a}$ Listed are adverse events with a possible immune-mediated cause and infusion reactions that occurred during study treatment or within the 90 days thereafter, regardless of attribution to study treatment or immune relatedness by the investigator. AE: Adverse event; ALT: alanine aminotransferase; AST: aspartate aminotransferase; Atezo: atezolizumab; Avel: avelumab; Axi: axitinib; Bev: bevacizumab; Cabo: cabozantinib; Evero: everolimus; Ipi: ipilimumab; Lenv: lenvatinib; Nivo: nivolumab; NR: not reported; PPE: palmar-plantar erythrodysthesia; Sun: sunitinib; -: not reported. 
two studies may be due to less robust antitumor activity of the anti-PD-1 backbone compared to nivolumab/ipilimumab and the increasing ability for patients with less aggressive tumors who progress on sunitinib to receive salvage anti-PD1 based immunotherapy. In contrast to the TFS period observed with nivolumab/ipilimumab, there is currently no evidence that anti-PD-1/VEGFR TKI combination regimens can produce continued response after cessation of the VEGFR TKI component. On the other hand, despite the fact that anti-PD-1/VEGFR TKI regimens have high rates of grade 3-4 adverse effects and frequent dose reductions, the Checkmate $9 \mathrm{ER}$ trial demonstrated significant improvement in health-related quality of life and burden of symptoms compared with sunitinib ${ }^{[66]}$. As data from several pivotal trials of anti-PD1/VEGFR TKI therapy continue to mature, their ability to produce improvements in late endpoints such as landmark PFS/OS and treatment free survival relative to nivolumab/ipilimumab will be essential to understanding their role as a front line therapy for patients with advanced ccRCC.

\section{Predictive biomarkers}

Although the MSKCC and IMDC models reliably predicted overall survival in patients receiving TKI therapy, there are currently no validated biomarkers of disease prognosis or prediction of response to ICI therapy or combination TKI/ICI therapy. Tumor PD-L1 expression was initially thought to be a promising biomarker given the observations that it is commonly overexpressed in $23 \%-56 \%$ of ccRCC tumors (depending on the assay) and is associated with poor outcomes ${ }^{[67,68]}$. However, the ability for $P D-L 1$ expression to reliably predict response to ICI has been inconsistent across studies. In Checkmate 214, patients with PD-L1 positive tumors treated with nivolumab/ipilimumab had improved PFS and OS compared to those with PD-L1 negative tumors. In the Keynote 426 study, patients responded to axitinib/pembrolizumab regardless of tumor PD-L1 status. Lastly, in the IMmotion 151 study patients with $\mathrm{PD}-\mathrm{L} 1$ negative disease appeared to have the most benefit from atezolizumab/bevacizumab relative to sunitinib. Taken together, PD-L1 positivity appears to predict better ORR on ICI therapy in patients, especially those aggressive disease; however, it remains limited in its ability to predict long-term PFS or OS benefits. PD-L1 expression appears to also predict lack of benefit from VEGFR TKI therapy alone, confounding its use as a biomarker in anti-PD1/VEGFR TKI combinations.

Acknowledging that most patients with ccRCC have PD-L1 negative tumors, it is important to recognize that the majority of responses to ICI will occur in the PD-L1 negative population. Although its role as a prognostic and predictive biomarker remains unclear, $P D-L 1$ expression remains an important risk stratification tool for clinical trials. Clearly additional biomarkers predictive of benefit for a particular regimen are needed to help with treatment choices in the current crowded first line therapeutic space.

Perhaps the most promising biomarkers have emerged from extensive genetic and gene expression profiling on tumors from the IMmotion 151 trial. Motzer et al. ${ }^{[69]}$ conducted integrated multi-omics evaluation of 823 tumors from patients with ccRCC and identified 7 distinct tumor molecular subsets (1) angiogenic/stromal; (2) angiogenic; (3) complement/oxidation; (4) T-effector/proliferative; (5) proliferative; (6) stromal/proliferative; and (7) SnoRNA ${ }^{[6]}$. The angiogenic groups 1 and 2 appeared to show benefit to both sunitinib and atezolizumab/bevacizumab. The proliferative groups 4-5 as well as group 7 appeared to show greater benefit to atezolizumab/bevacizumab compared to sunitinib alone. Groups 3 and 6 did not appear to benefit from either treatment approach. Lastly, somatic mutations in PBRM1 and KDM5C associate with high angiogenesis and AMPK fatty acid oxidation suggesting benefits from angiogenesis blockade ${ }^{[70]}$. Whereas $C D K N_{2} A, B A P 1$, and TP53 appear to associate with increase cell cycle and anabolic metabolism suggesting benefit from ICI therapies ${ }^{[7]}$. Taken together these discoveries, if validated using contemporary FDA approved regimens, could be used to categorize patients based on genetic and gene expression profiles and thus determine the optimal treatment approach (e.g., combination ICI, combination VEGFR TKI/ICI, or novel agents) while also mitigating drug and financial toxicities ${ }^{[72]}$. Importantly, such validation studies 
for these and other predictive biomarkers should be linked to goals of care including novel endpoints mentioned above such as landmark OS and PFS, TFS and QOL throughout to the TFS period which better reflect the impact of the immunotherapy component.

\section{FUTURE DIRECTIONS}

The role of cytoreductive nephrectomy, which previously provided both symptomatic benefit and improved overall survival in combination with cytokine therapies, is yet to be defined with current ICI and ICI/VEGFR TKI combinations ${ }^{[73-76]}$. The NORDIC-SUN (NCT03977571) and CYTOSHRINK (NCT04090710) trials are evaluating induction nivolumab/ipilimumab followed by delayed cytoreductive nephrectomy or interim stereotactic body radiation, respectively followed by nivolumab maintenance ${ }^{[77,78]}$ [Table 4]. The Phase 2 Cyto-KIK trial (NCT04322955) is evaluating induction nivolumab/cabozantinib with delayed cytoreductive nephrectomy followed by resumption of systemic therapy ${ }^{[79]}$. Lastly, the phase 3 PROBE trial (NCT04510597) includes checkpoint inhibitor-based induction with nivolumab/ipilimumab or axitinib/pembrolizumab followed by randomization to nephrectomy or continuation of systemic therapy for patients with $\mathrm{PR} / \mathrm{SD}$ and discontinuation of study for patients with $\mathrm{CR} / \mathrm{PD}^{[80]}$. Each of these studies will collect vital clinicopathologic data that have the potential to our understanding of the biologic processes underlying responses to therapy and guide initial and subsequent treatment choices.

There are several ongoing studies of doublet and triplet regimens for advanced treatment naïve ccRCC. For patients with intermediate/poor risk disease, the phase 3 trials Checkmate 209-8Y8 (NCT03873402) and COSMIC 313 (NCT03937219) are investigating nivolumab/ipilimumab compared to nivolumab alone and nivolumab/ipilimumab/cabozantinib, respectively with primary endpoints of PFS by central review ${ }^{[54,81]}$ [Table 4]. The PD1GREE (NCT03793166) phase 3 adaptive trial for patients with intermediate/poor risk treatment naïve ccRCC compares induction nivolumab/ipilimumab followed by either nivolumab/cabozantinib or nivolumab alone for patients with PR or SD at 12 weeks $^{[82]}$. Following induction nivolumab/ipilimumab, patients with CR continue on nivolumab maintenance, whereas patients with PD change to cabozantinib monotherapy. However, there remains an unmet need for a clinical trial comparing first line nivolumab/ipilimumab to anti-PD1/VEGF therapy for all risk disease patients with endpoints of landmark PFS and OS, complete response rates, treatment free survival, and quality of life. Optimally such a trial could also validate some of the intriguing biomarker data emerging from some of the recently published trials. With a widening landscape of treatment combinations, clinical trials investigating sequences and combinations of therapies must be designed to demonstrate impact on long-term outcomes such as complete responses, landmark PFS/OS, treatment free survival, and quality of life.

\section{CONCLUSION}

The first line treatment paradigm for advanced ccRCC has rapidly evolved with an expanding number of combination anti-angiogenic/PD1/L1 regimens including axitinib/pembrolizumab, axitinib/avelumab, cabozantinib/nivolumab and likely lenvatinib/pembrolizumab being added to the existing VEGFR TKI monotherapy and ICI combination regimens. Figure 1 depicts a current treatment algorithm for frontline therapy of patients with advanced ccRCC. All of these anti-angiogenic/anti-PD1/L1 combinations have been (or will be) approved based on benefits relative to sunitinib. These promising early responses must be contextualized to the long-term benefits of dual immune checkpoint blockade with nivolumab/ipilimumab. Indeed, the question of whether antiangiogenic and ICI therapies provide synergistic, additive or subadditive effects on long-term outcomes, such as cure rate, treatment free survival and landmark PFS and OS remains under intense scrutiny. Surveillance of long-term toxicities and quality of life measures will also be important considerations. Prospectively validated biomarkers will be essential with the potential to match individual patients' disease biology with checkpoint inhibitors, antiangiogenic TKI, or novel therapies to 
Table 4. Current clincial trails for first line ccRCC

\begin{tabular}{|c|c|c|c|c|c|}
\hline $\begin{array}{c}\text { Title } \\
\text { Systemic therapy }\end{array}$ & $\begin{array}{l}\text { Study } \\
\text { design }\end{array}$ & Patients & Regimen & Study details & $\begin{array}{c}\text { Key results/completion } \\
\text { status }\end{array}$ \\
\hline $\begin{array}{l}\text { Checkmate 8Y8 } \\
(\text { NCT03873402) }\end{array}$ & $\begin{array}{l}1 \mathrm{~L}, \text { Phase } \\
3\end{array}$ & $\begin{array}{l}\text { Int/poor risk ( } N \\
=418)\end{array}$ & $\begin{array}{l}\text { Nivo } \\
\text { Nivo/Ipi }\end{array}$ & $\begin{array}{l}\text { Co-primary endpoint: PFS } \\
\text { and ORR by BICR }\end{array}$ & $\begin{array}{l}\text { Estimated study completion: } \\
\text { date April } 2025\end{array}$ \\
\hline $\begin{array}{l}\text { COSMIC } 313 \\
(\text { NCT03937219) }\end{array}$ & $\begin{array}{l}1 \mathrm{~L} \text {, Phase } \\
3\end{array}$ & $\begin{array}{l}\text { Int/poor risk ( } N \\
=840)\end{array}$ & $\begin{array}{l}\text { Cabo/Nivo } \\
\text { Cabo/Nivo/lpi }\end{array}$ & $\begin{array}{l}\text { Primary endpoint: duration } \\
\text { PFS by BICR }\end{array}$ & $\begin{array}{l}\text { Estimated study completion } \\
\text { date: March } 2025\end{array}$ \\
\hline $\begin{array}{l}\text { PD1GREE } \\
\left(_{(N C T 03793166)^{[82]}}\right.\end{array}$ & $\begin{array}{l}1 \mathrm{~L} \text {, Phase } \\
3\end{array}$ & $\begin{array}{l}\text { Int/poor risk (N } \\
=1046)\end{array}$ & $\begin{array}{l}\text { Nivo/Ipi induction, } \\
\text { Nivo } \\
\text { Nivo/Cabo }\end{array}$ & Primary endpoint: OS & $\begin{array}{l}\text { Estimated study completion } \\
\text { date: April } 2022\end{array}$ \\
\hline \multicolumn{6}{|l|}{ Surgery/radiation } \\
\hline $\begin{array}{l}\text { NORDIC-SUN } \\
\left(_{(\text {NCTO3977571) }}^{[77]}\right.\end{array}$ & $\begin{array}{l}1 \mathrm{~L}, \text { Phase } \\
3\end{array}$ & $\begin{array}{l}\text { Int/poor risk ( } N \\
=400)\end{array}$ & $\begin{array}{l}\text { Nivo } 3 \mathrm{mg} / \mathrm{kg} / \mathrm{lpi} \\
1 \mathrm{mg} / \mathrm{kg} \text { q3wk x4 } \\
\text { - Delayed CRN/Nivo } \\
\text { - Nivo }\end{array}$ & Primary endpoint: OS & $\begin{array}{l}\text { Estimated study completion } \\
\text { date: September } 2025\end{array}$ \\
\hline $\begin{array}{l}\text { CYTOSHRINK } \\
(\text { NCT04090710 })^{[78]}\end{array}$ & $\begin{array}{l}1 \mathrm{~L}, \text { Phase } \\
2\end{array}$ & $\begin{array}{l}\text { Int/poor risk (N } \\
=78)\end{array}$ & $\begin{array}{l}\text { Nivo } 3 \text { mg/kg/lpi } 1 \\
\text { mg/kg q3wk x4 } \\
\text { - SBRT prior to cycle } 2 \\
\text { - no SBRT }\end{array}$ & Primary endpoint: PFS & $\begin{array}{l}\text { Estimated study completion } \\
\text { date: April } 2022\end{array}$ \\
\hline $\begin{array}{l}\text { Cyto-KIK trial } \\
\left(^{\text {NCT04322955) }}\right)^{[79]}\end{array}$ & $\begin{array}{l}\text { 1L, Phase } \\
2\end{array}$ & $\begin{array}{l}\text { Treatment naive } \\
(N=48)\end{array}$ & $\begin{array}{l}\text { Nivo } 480 \mathrm{mg} \\
\text { q4wk/Cabo } 40 \mathrm{mg} \text { qd } \\
\text { - Delayed CRN }\end{array}$ & $\begin{array}{l}\text { Primary endpoint: \% of } \\
\text { participants with a complete } \\
\text { response }\end{array}$ & $\begin{array}{l}\text { Estimated study completion } \\
\text { date: February } 2022\end{array}$ \\
\hline $\begin{array}{l}\text { PROBE trial } \\
\left(^{(\text {NCT04510597) }}\right)^{[80]}\end{array}$ & $\begin{array}{l}\text { 1L, Phase } \\
3\end{array}$ & $\begin{array}{l}\text { Treatment naive } \\
(N=364)\end{array}$ & $\begin{array}{l}\text { Initial CRN then } \\
\text { systemic therapy } \\
\text { Systemic therapy } \\
\text { alone }\end{array}$ & Primary endpoint: OS & $\begin{array}{l}\text { Estimated study completion } \\
\text { date: July } 2033\end{array}$ \\
\hline
\end{tabular}

Cabo: Cabozantinib; CRN: cytoreductive nephrectomy; IMDC: International Metastatic Renal Cell Carcinoma; int: intermediate; Ipi: ipilimumab; Nivo: nivolumab; ORR: objective response rate; OS: overall survival; PFS: progression free survival; Ph: phase; q3wk: every 3 weeks; qd: once daily; wk: week; SBRT: stereotactic body radiation therapy.

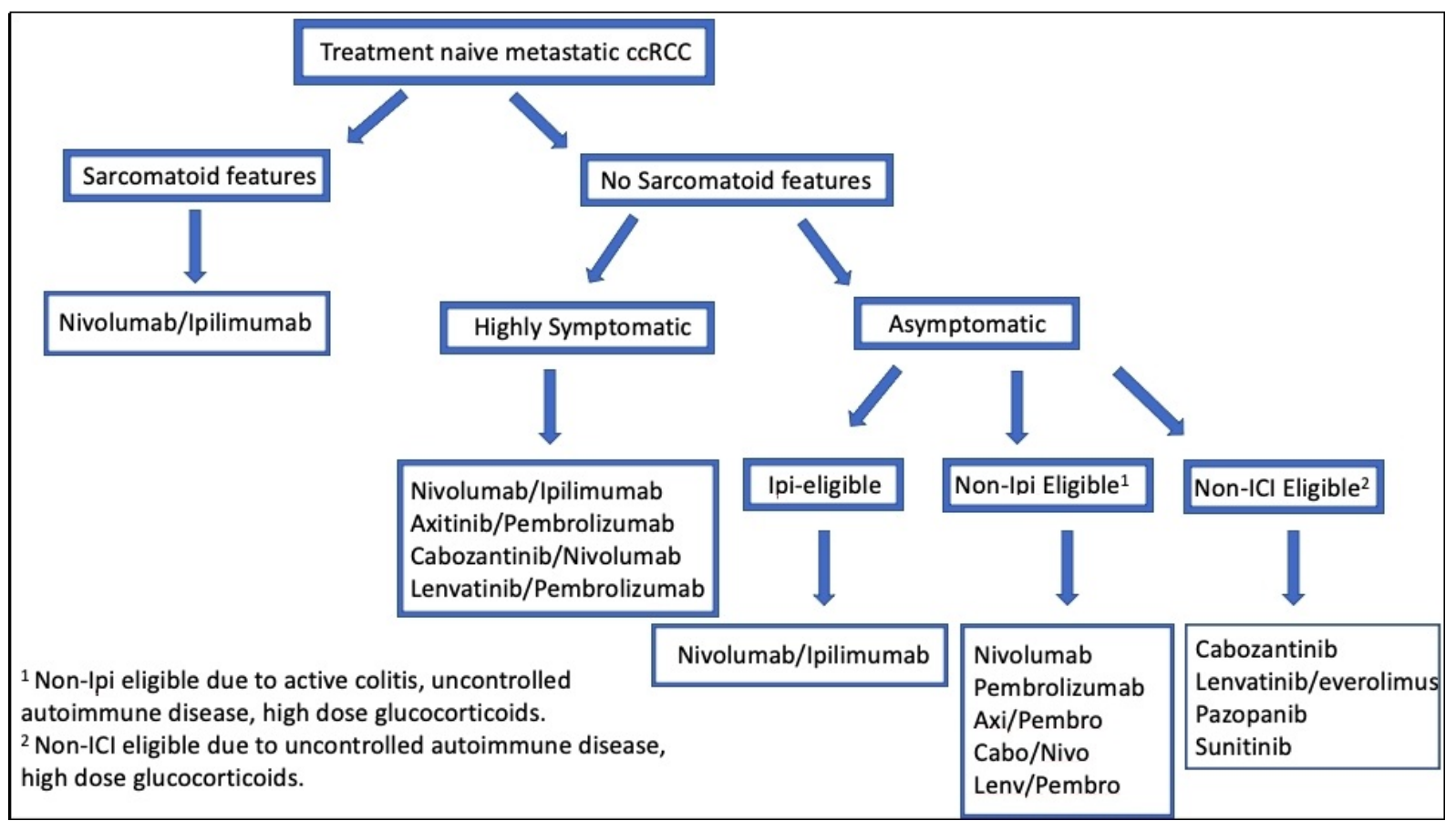

Figure 1. Treatment algorithm for treatmnet naïve metastatic ccRCC. ccRCC: Clear cell renal cell carcinoma. 
guide first line and sequential treatment strategies.

\section{DECLARATIONS}

\section{Authors' contributions}

Responsible for the paper: Atkins MB

Concept, design, definition of intellectual content: Atkins MB

Literature search: Serzan M, Atkins MB

Manuscript preparation: Serzan M, Atkins MB

Manuscript editing: Serzan M, Atkins MB

Manuscript revision: Serzan M, Atkins MB

\section{Availability of data and materials}

Not applicable.

\section{Financial support and sponsorship}

None.

\section{Conflicts of interest}

Both authors declared that there are no conflicts of interest.

\section{Ethical approval and consent to participate}

Not applicable.

\section{Consent for publication}

Not applicable.

\section{Copyright}

(c) The Author(s) 2021.

\section{REFERENCES}

1. Ferlay J, Colombet M, Soerjomataram I, et al. Estimating the global cancer incidence and mortality in 2018: GLOBOCAN sources and methods. Int J Cancer 2019;144:1941-53. DOI PubMed

2. Padala SA, Barsouk A, Thandra KC, et al. Epidemiology of Renal Cell Carcinoma. World J Oncol 2020;11:79-87. DOI PubMed PMC

3. Sorbellini M, Kattan MW, Snyder ME, et al. A postoperative prognostic nomogram predicting recurrence for patients with conventional clear cell renal cell carcinoma. J Urol 2005;173:48-51. DOI PubMed

4. Surveillance, Epidemiology, and End Results Program, National Cancer Institute. Cancer stat facts: kidney and renal pelvis cancer. Available from: https://seer.cancer.gov/statfacts/html/kidrp.html [Last accessed on 21 May 2021].

5. Dizman N, Philip EJ, Pal SK. Genomic profiling in renal cell carcinoma. Nat Rev Nephrol 2020;16:435-51. DOI PubMed

6. Ricketts CJ, De Cubas AA, Fan H, et al; Cancer Genome Atlas Research Network. The cancer genome atlas comprehensive molecular characterization of renal cell carcinoma. Cell Rep 2018;23:3698. DOI PubMed

7. Nickerson ML, Jaeger E, Shi Y, et al. Improved identification of von Hippel-Lindau gene alterations in clear cell renal tumors. Clin Cancer Res 2008;14:4726-34. DOI PubMed PMC

8. Shen C, Kaelin WG Jr. The VHL/HIF axis in clear cell renal carcinoma. Semin Cancer Biol 2013;23:18-25. DOI PubMed PMC

9. Gossage L, Eisen T, Maher ER. VHL, the story of a tumour suppressor gene. Nat Rev Cancer 2015;15:55-64. DOI PubMed

10. Motzer RJ, Hutson TE, Tomczak P, et al. Overall survival and updated results for sunitinib compared with interferon alfa in patients with metastatic renal cell carcinoma. J Clin Oncol 2009;27:3584-90. DOI PubMed PMC

11. Sternberg CN, Davis ID, Mardiak J, et al. Pazopanib in locally advanced or metastatic renal cell carcinoma: results of a randomized phase III trial. J Clin Oncol 2010;28:1061-8. DOI PubMed

12. Motzer RJ, Hutson TE, Cella D, et al. Pazopanib versus sunitinib in metastatic renal-cell carcinoma. $N$ Engl J Med 2013;369:722-31. DOI PubMed

13. Choueiri TK, Halabi S, Sanford BL, et al. Cabozantinib versus sunitinib as initial targeted therapy for patients with metastatic renal cell carcinoma of poor or intermediate risk: the alliance A031203 CABOSUN trial. J Clin Oncol 2017;35:591-7. DOI PubMed PMC

14. Iyer G, Hanrahan AJ, Milowsky MI, et al. Genome sequencing identifies a basis for everolimus sensitivity. Science 2012;338:221. 
DOI PubMed PMC

15. Motzer RJ, Escudier B, McDermott DF, et al; CheckMate 025 investigators. Nivolumab versus everolimus in advanced renal-cell carcinoma. N Engl J Med 2015;373:1803-13. DOI PubMed PMC

16. Motzer RJ, Tannir NM, McDermott DF, et al; CheckMate 214 Investigators. Nivolumab plus Ipilimumab versus sunitinib in advanced renal-cell carcinoma. N Engl J Med 2018;378:1277-90. DOI PubMed PMC

17. Heng DY, Xie W, Regan MM, et al. External validation and comparison with other models of the International Metastatic Renal-Cell Carcinoma Database Consortium prognostic model: a population-based study. Lancet Oncol 2013;14:141-8. DOI PubMed PMC

18. Motzer RJ, Bacik J, Murphy BA, Russo P, Mazumdar M. Interferon-alfa as a comparative treatment for clinical trials of new therapies against advanced renal cell carcinoma. J Clin Oncol 2002;20:289-96. DOI PubMed

19. Jubb AM, Pham TQ, Hanby AM, et al. Expression of vascular endothelial growth factor, hypoxia inducible factor 1alpha, and carbonic anhydrase IX in human tumours. J Clin Pathol 2004;57:504-12. DOI PubMed PMC

20. Goel S, Duda DG, Xu L, et al. Normalization of the vasculature for treatment of cancer and other diseases. Physiol Rev 2011;91:1071121. DOI PubMed PMC

21. Sennino B, McDonald DM. Controlling escape from angiogenesis inhibitors. Nat Rev Cancer 2012;12:699-709. DOI PubMed PMC

22. Lai Y, Zhao Z, Zeng T, et al. Crosstalk between VEGFR and other receptor tyrosine kinases for TKI therapy of metastatic renal cell carcinoma. Cancer Cell Int 2018;18:31. DOI PubMed PMC

23. Escudier B, Porta C, Bono P, et al. Randomized, controlled, double-blind, cross-over trial assessing treatment preference for pazopanib versus sunitinib in patients with metastatic renal cell carcinoma: PISCES study. J Clin Oncol 2014;32:1412-8. DOI PubMed

24. Hudes G, Carducci M, Tomczak P, et al; Global ARCC Trial. Temsirolimus, interferon alfa, or both for advanced renal-cell carcinoma. N Engl J Med 2007;356:2271-81. DOI PubMed

25. Motzer RJ, Barrios CH, Kim TM, et al. Phase II randomized trial comparing sequential first-line everolimus and second-line sunitinib versus first-line sunitinib and second-line everolimus in patients with metastatic renal cell carcinoma. J Clin Oncol 2014;32:2765-72. DOI PubMed PMC

26. Motzer RJ, Jonasch E, Boyle S, et al. NCCN Guidelines Insights: Kidney Cancer, Version 1.2021. J Natl Compr Canc Netw 2020;18:1160-70. DOI PubMed

27. Zhou L, Liu XD, Sun M, et al. Targeting MET and AXL overcomes resistance to sunitinib therapy in renal cell carcinoma. Oncogene 2016;35:2687-97. DOI PubMed PMC

28. Choueiri TK, Hessel C, Halabi S, et al. Cabozantinib versus sunitinib as initial therapy for metastatic renal cell carcinoma of intermediate or poor risk (Alliance A031203 CABOSUN randomised trial): progression-free survival by independent review and overall survival update. Eur J Cancer 2018;94:115-25. DOI PubMed PMC

29. Motzer R, Alekseev B, Rha SY, et al; CLEAR Trial Investigators. Lenvatinib plus Pembrolizumab or Everolimus for Advanced Renal Cell Carcinoma. N Engl J Med 2021;384:1289-300. DOI PubMed

30. Hutson TE, Lesovoy V, Al-shukri S, et al. Axitinib versus sorafenib as first-line therapy in patients with metastatic renal-cell carcinoma: a randomised open-label phase 3 trial. Lancet Oncol 2013;14:1287-94. DOI PubMed

31. Motzer RJ, Nosov D, Eisen T, et al. Tivozanib versus sorafenib as initial targeted therapy for patients with metastatic renal cell carcinoma: results from a phase III trial. J Clin Oncol 2013;31:3791-9. DOI PubMed PMC

32. Motzer RJ, Escudier B, Tomczak P, et al. Axitinib versus sorafenib as second-line treatment for advanced renal cell carcinoma: overall survival analysis and updated results from a randomised phase 3 trial. Lancet Oncol 2013;14:552-62. DOI PubMed

33. Rini BI, Pal SK, Escudier BJ, et al. Tivozanib versus sorafenib in patients with advanced renal cell carcinoma (TIVO-3): a phase 3, multicentre, randomised, controlled, open-label study. Lancet Oncol 2020;21:95-104. DOI PubMed

34. McDermott DF, Cheng SC, Signoretti S, et al. The high-dose aldesleukin "select" trial: a trial to prospectively validate predictive models of response to treatment in patients with metastatic renal cell carcinoma. Clin Cancer Res 2015;21:561-8. DOI PubMed PMC

35. Coppin C, Porzsolt F, Autenrieth M, et al. Immunotherapy for advanced renal cell cancer. Cochrane database of systematic reviews. Chichester: John Wiley \& Sons, Ltd; 1996.

36. Blankenstein T, Coulie PG, Gilboa E, Jaffee EM. The determinants of tumour immunogenicity. Nat Rev Cancer 2012;12:307-13. DOI PubMed PMC

37. Kopecky O, Lukesová S, Vroblová V, et al. Phenotype analysis of tumour-infiltrating lymphocytes and lymphocytes in peripheral blood in patients with renal carcinoma. Acta Medica (Hradec Kralove) 2007;50:207. PubMed

38. Komohara Y, Hasita H, Ohnishi K, et al. Macrophage infiltration and its prognostic relevance in clear cell renal cell carcinoma. Cancer Sci 2011;102:1424-31. DOI PubMed

39. Chen DS, Mellman I. Oncology meets immunology: the cancer-immunity cycle. Immunity 2013;39:1-10. DOI PubMed

40. Walker LS, Sansom DM. The emerging role of CTLA4 as a cell-extrinsic regulator of T cell responses. Nat Rev Immunol 2011;11:852-63. DOI PubMed

41. Dong Y, Sun Q, Zhang X. PD-1 and its ligands are important immune checkpoints in cancer. Oncotarget 2017;8:2171. DOI PubMed PMC

42. Hammers HJ, Plimack ER, Infante JR, et al. Safety and efficacy of nivolumab in combination with ipilimumab in metastatic renal cell carcinoma: the CheckMate 016 study. J Clin Oncol 2017;35:3851-8. DOI PubMed PMC

43. Motzer RJ, Escudier B, McDermott DF, et al. Survival outcomes and independent response assessment with nivolumab plus ipilimumab versus sunitinib in patients with advanced renal cell carcinoma: 42-month follow-up of a randomized phase 3 clinical trial. J Immunother Cancer 2020;8:e00891. DOI PubMed PMC

44. Tannir NM, McDermott DF, Escudier B, Hammers HJ, et al. Overall survival and independent review of response in CheckMate 214 
with 42-month follow-up: first-line nivolumab + ipilimumab (N+I) versus sunitinib (S) in patients (pts) with advanced renal cell carcinoma (aRCC). J Clin Oncol 2020;38:6_suppl, 609. DOI

45. Tannir NM, Signoretti S, Choueiri TK, et al. Efficacy and Safety of Nivolumab Plus Ipilimumab versus Sunitinib in First-line Treatment of Patients with Advanced Sarcomatoid Renal Cell Carcinoma. Clin Cancer Res 2021;27:78-86. DOI PubMed

46. Tykodi SS, Donskov F, Lee JL, Szczylik C, Malik J et al. First-line pembrolizumab (pembro) monotherapy in advanced clear cell renal cell carcinoma (ccRCC): updated results for KEYNOTE-427 cohort A. J Clin Oncol 2019;37:15_suppl, 4570. DOI

47. Atkins MB, Jegede $\mathrm{O}$, Haas NB, et al. Phase II study of nivolumab and salvage nivolumab + ipilimumab in treatment-naïve patients (pts) with advanced renal cell carcinoma (RCC) (HCRN GU16-260). J Clin Oncol 2020;38(15_suppl):5006. DOI

48. US National Library of Medicine. Available from: https://clinicaltrials.gov/ct2/show/NCT03873402 (2021) [Last accessed on 21 May 2021].

49. Guislain A, Gadiot J, Kaiser A, et al. Sunitinib pretreatment improves tumor-infiltrating lymphocyte expansion by reduction in intratumoral content of myeloid-derived suppressor cells in human renal cell carcinoma. Cancer Immunol Immunother 2015;64:124150. DOI PubMed

50. Kwilas AR, Ardiani A, Donahue RN, Aftab DT, Hodge JW. Dual effects of a targeted small-molecule inhibitor (cabozantinib) on immune-mediated killing of tumor cells and immune tumor microenvironment permissiveness when combined with a cancer vaccine. $J$ Transl Med 2014;12:294. DOI PubMed PMC

51. Li YL, Zhao H, Ren XB. Relationship of VEGF/VEGFR with immune and cancer cells: staggering or forward? Cancer Biol Med 2016;13:206-14. DOI PubMed PMC

52. Kwilas AR, Donahue RN, Tsang KY, Hodge JW. Immune consequences of tyrosine kinase inhibitors that synergize with cancer immunotherapy. Cancer Cell Microenviron 2015;2:e677. DOI PubMed PMC

53. Zhang Q, Tian K, Xu J, et al. Synergistic Effects of Cabozantinib and EGFR-Specific CAR-NK-92 Cells in Renal Cell Carcinoma. $J$ Immunol Res 2017;2017:6915912. DOI PubMed PMC

54. Bao Y, Wang Z, Liu B, et al. A feed-forward loop between nuclear translocation of CXCR4 and HIF-1 $\alpha$ promotes renal cell carcinoma metastasis. Oncogene 2019;38:881-95. DOI PubMed PMC

55. Liu XD, Hoang A, Zhou L, et al. Resistance to Antiangiogenic Therapy Is Associated with an Immunosuppressive Tumor Microenvironment in Metastatic Renal Cell Carcinoma. Cancer Immunol Res 2015;3:1017-29. DOI PubMed PMC

56. Hipp MM, Hilf N, Walter S, et al. Sorafenib, but not sunitinib, affects function of dendritic cells and induction of primary immune responses. Blood 2008;111:5610-20. DOI PubMed

57. Amin A, Plimack ER, Ernstoff MS, et al. Safety and efficacy of nivolumab in combination with sunitinib or pazopanib in advanced or metastatic renal cell carcinoma: the CheckMate 016 study. J Immunother Cancer 2018;6:109. DOI PubMed PMC

58. Rini BI, Powles T, Atkins MB, et al. Atezolizumab plus bevacizumab versus sunitinib in patients with previously untreated metastatic renal cell carcinoma (IMmotion151): a multicentre, open-label, phase 3, randomised controlled trial. Lancet 2019;393:2404-15. DOI PubMed

59. Rini BI, Plimack ER, Stus V, et al; KEYNOTE-426 Investigators. Pembrolizumab plus axitinib versus sunitinib for advanced renalcell carcinoma. N Engl J Med 2019;380:1116-27. DOI PubMed

60. Powles T, Plimack ER, Soulières D, Waddell T, Stus V et al. Pembrolizumab plus axitinib versus sunitinib monotherapy as first-line treatment of advanced renal cell carcinoma (KEYNOTE-426): extended follow-up from a randomized, open-label, phase 3 trial. Lancet Oncol 2020;21:1563-73. DOI PubMed

61. Motzer RJ, Penkov K, Haanen J, et al. Avelumab plus Axitinib versus Sunitinib for Advanced Renal-Cell Carcinoma. N Engl J Med 2019;380:1103-15. DOI PubMed PMC

62. Choueiri TK, Motzer RJ, Rini BI, et al. Updated efficacy results from the JAVELIN Renal 101 trial: first-line avelumab plus axitinib versus sunitinib in patients with advanced renal cell carcinoma. Ann Oncol 2020;31:1030-9. DOI PubMed

63. Choueiri TK, Powles T, Burotto M, et al; CheckMate 9ER Investigators. Nivolumab plus cabozantinib versus sunitinib for advanced renal-cell carcinoma. N Engl J Med 2021;384:829-41. DOI PubMed

64. Regan MM, Atkins MB, Powles T, Werner L, Mantia C et al. Treatment-free survival, with and without toxicity, as a novel outcome applied to immuno-oncology agents in advanced renal cell carcinoma. Ann Oncol 2019;30:v393-4. DOI

65. Regan M, Jegede OA, Mantia C, Powles T, Werner L et al. 713P Treatment-free survival, with and without toxicity, after immunooncology vs targeted therapy for advanced renal cell carcinoma (aRCC): 42-month results of CheckMate 214. Ann Oncol 2020;31:S561. DOI

66. Cella D, Grünwald V, Escudier B, et al. Patient-reported outcomes of patients with advanced renal cell carcinoma treated with nivolumab plus ipilimumab versus sunitinib (CheckMate 214): a randomised, phase 3 trial. Lancet Oncol 2019;20:297-310. DOI PubMed PMC

67. Leite KR, Reis ST, Junior JP, et al. PD-L1 expression in renal cell carcinoma clear cell type is related to unfavorable prognosis. Diagn Pathol 2015;10:189. DOI PubMed PMC

68. Iacovelli R, Nolè F, Verri E, et al. Prognostic Role of PD-L1 Expression in Renal Cell Carcinoma. A Systematic Review and MetaAnalysis. Target Oncol 2016;11:143-8. DOI PubMed

69. Motzer RJ, Banchereau R, Hamidi H, et al. Molecular Subsets in Renal Cancer Determine Outcome to Checkpoint and Angiogenesis Blockade. Cancer Cell 2020;38:803-17.e4. DOI PubMed

70. Kapur P, Christie A, Rajaram S, Brugarolas J. What morphology can teach us about renal cell carcinoma clonal evolution. Kidney Cancer J 2020;18:68-75.

71. Gu YF, Cohn S, Christie A, et al. Modeling renal cell carcinoma in mice: Bap1 and Pbrm1 inactivation drive tumor grade. Cancer 
Discov 2017;7:900-17. DOI PubMed PMC

72. Brugarolas J, Rajaram S, Christie A, Kapur P. The Evolution of Angiogenic and Inflamed Tumors: The Renal Cancer Paradigm. Cancer Cell 2020;38:771-3. DOI PubMed

73. Flanigan RC, Salmon SE, Blumenstein BA, et al. Nephrectomy followed by interferon alfa- $2 \mathrm{~b}$ compared with interferon alfa- $2 \mathrm{~b}$ alone for metastatic renal-cell cancer. N Engl J Med 2001;345:1655-9. DOI PubMed

74. Mickisch G, Garin A, van Poppel H, de Prijck L, Sylvester R. Radical nephrectomy plus interferon-alfa-based immunotherapy compared with interferon alfa alone in metastatic renal-cell carcinoma: a randomised trial. Lancet 2001;358:966-70. DOI PubMed

75. Méjean A, Ravaud A, Thezenas S, et al. Sunitinib Alone or after Nephrectomy in Metastatic Renal-Cell Carcinoma. N Engl J Med 2018;379:417-27. DOI PubMed

76. Bex A, Mulders P, Jewett M, et al. Comparison of Immediate vs Deferred Cytoreductive Nephrectomy in Patients With Synchronous Metastatic Renal Cell Carcinoma Receiving Sunitinib: The SURTIME Randomized Clinical Trial. JAMA Oncol 2019;5:164-70. DOI PubMed PMC

77. US National Library of Medicine. Available from: https://clinicaltrials.gov/ct2/show/NCT03977571 (2021) [Last accessed on 21 May 2021].

78. US National Library of Medicine. Available from: https://clinicaltrials.gov/ct2/show/NCT04090710 (2021) [Last accessed on 21 May 2021].

79. US National Library of Medicine. Available from: https://clinicaltrials.gov/ct2/show/NCT04322955 (2021) [Last accessed on 21 May 2021].

80. US National Library of Medicine. Available from: https://clinicaltrials.gov/ct2/show/NCT04510597 (2021) [Last accessed on 21 May 2021].

81. US National Library of Medicine. Available from: https://clinicaltrials.gov/ct2/show/NCT03937219 (2021) [Last accessed on 21 May 2021].

82. US National Library of Medicine. Available from: https://clinicaltrials.gov/ct2/show/NCT03793166 (2021) [Last accessed on 21 May 2021]. 